\title{
Modal expansion approach to optical-frequency-comb generation with monolithic whispering-gallery-mode resonators
}

\author{
Yanne K. Chembo* and Nan Yu \\ Jet Propulsion Laboratory, California Institute of Technology, 4800 Oak Grove Drive, Pasadena, California 91109, USA
}

(Received 6 April 2010; published 7 September 2010)

\begin{abstract}
We describe a general framework based on modal expansion for the study of optical-frequency combs generated with monolithic whispering-gallery-mode resonators. We obtain a set of time-domain rate equations describing the dynamics of each mode as a function of the main characteristics of the cavity, namely, Kerr nonlinearity, absorption, coupling losses, and cavity dispersion (geometrical and material). A stability analysis of the various side modes is performed, which finds analytically the threshold power needed for comb generation. We show that the various whispering gallery modes are excited in a nontrivial way, strongly dependent on the value of the overall cavity dispersion. We demonstrate that the combs are not simply generated through a direct transfer of energy from the pumped mode to all their neighbors but rather through complex intermediate interactions. Anomalous cavity dispersion is also demonstrated to be critical for these cascading processes, and comb generation is thereby unambiguously linked to modulational instability. This theory accurately describes the emergence of spectral modulation and free spectral-range tunability in the comb. It also enables a clear understanding of the various phenomena responsible for the spectral span limitation. Our theoretical predictions are in excellent agreement with the numerical simulations, and they successfully explain the internal mechanisms responsible for the generation of hundreds of Kerr modes in monolithic whispering-gallery-mode resonators.
\end{abstract}

DOI: 10.1103/PhysRevA.82.033801

PACS number(s): 42.62.Eh, 42.65.Hw, 42.65.Ky, 42.65.Sf

\section{INTRODUCTION}

Optical-frequency combs are sets of regularly spaced spectral lines in the ultraviolet, visible, or infrared ranges. They have for long been generated with mode-locked ultrafast lasers, as periodic trains of ultrashort laser pulses yield such equidistant lines in the spectral domain. When their frequency span covers one octave, the combs can be autoreferenced, and it is therefore possible to transfer the metrologic precision of optical laser frequencies down to the $\mathrm{GHz}$ or $\mathrm{THz}$ ranges (see Refs. [1-3], and references therein). Many applications can benefit from these combs: fundamental physics, time-frequency metrology, navigation systems, spectroscopy, sensing, or ultralow phase noise microwave and terahertz generation.

An interesting method has been demonstrated recently for the generation of these combs, and it relies on the hyperparametric excitation of the whispering gallery modes (WGMs) of an ultrahigh $Q$ monolithic resonator [4-7]. In this configuration, the dielectric microresonator is shaped as a cylinder, a sphere, or a toroid whose principal dimension ranges from a few tens of micrometers to few millimeters (see review articles [8-10], and references therein). Provided that the bulk material is low loss and the resonator has smooth surfaces (subnanometer surface irregularities), the light can be trapped for few microseconds by total internal reflection. Their free-spectral range (FSR) may vary from a few gigahertz to a few terahertz, depending on the resonator's radius, and their quality factor $Q$ can be exceptionally high, of the order of $10^{10}$ [11]. In these resonators, the small volume of

*yanne.chembo@jpl.nasa.gov; Present address: Optics Department, FEMTO-ST Institute, UMR CNRS 6174, 16 Route de Gray, F-25030 Besançon CEDEX, France. confinement, high photon density, and long photon storage time (proportional to the quality factor $Q$ ) induce a very strong light-matter interaction. Depending on the dielectric material, this strong coupling can generate a highly efficient four-wave mixing (FWM), where two pump photons are transformed into two sideband photons through the Kerr nonlinearity. Provided that the pump is powerful enough, an optical-frequency comb, sometimes referred to as a Kerr comb [4], is generated through a cascaded creation of such sideband photons, resulting from a huge sum of weighted interactions involving any four photons fulfilling energy and angular momentum conservation requirements $[4,12,13]$. The essential advantages of this method for comb generation are intrinsic simplicity, small size, and very low power consumption.

There have been noteworthy contributions to the theoretical understanding of optical-frequency comb generation in WGM resonators. The pioneering articles on this topic mainly focused on sideband parametric generation, where the pump excites a signal and an idler side mode through degenerate four-wave mixing. Along this line, threshold conditions were analyzed and discussed by several authors [14-16], and the effect of dispersion on parametric sideband generation was investigated in Ref. [17]. As far as wide-span WGM-comb generation is concerned, an interesting study was reported in Ref. [18], and the authors proposed a fully numerical approach where the laser light beam in the resonator was treated as if propagating along an unfolded periodic trajectory.

These previous works provide some understanding of comb generation with WGM resonators. However, there are still several important issues that need to be addressed. For example, there are some hints about the physical phenomena limiting the spectral span of the comb, but the lack of a coherent theoretical background does not give any indication about their relative importance. Along the same line, the role of 
cavity dispersion is recognized as critical, but no one knows exactly to which extent. Even the issue of equidistance is wide open because to the best of our knowledge, there is no theoretical insight about the importance of degenerate FWM (leading to only pairwise equidistance) relative to nondegenerate FWM (ensuring comblike equidistance). No stability diagram has ever been proposed for WGM combs so that the conditions under which they are stable and stationary are unknown. It is also interesting to note that recently, a very important phenomenon-here referred to as versatilityhas been experimentally demonstrated in these WGM comb generators. It relates to the capacity for the system to generate various combs whose FSRs are integer multiples of the cavity FSR. The spectral periodicity of the comb can be tuned through a frequency shift of the pumping laser [6] or a variation of the coupling strength [7]. This phenomenon remains unexplained.

Our aim in this article is to describe a coherent theoretical framework based on modal expansion in order to address the aforementioned open issues. In particular, we will use the nonlinear dynamics formalism to investigate the interaction between nonlinearity, $Q$ factor of the resonator, and cavity dispersion as well as the spectral profile and the temporal dynamics of the comb. This article also presents the explicit demonstration of the theoretical results reported in Ref. [19].

The outline of the article is as follows. The system under study is presented in Sec. II. The following three sections are devoted to the introduction of the multimode approach used in our theoretical analysis, the analytical determination of the WGMs, and the explicit derivation of the modal equations, respectively. Then we perform in Sec. VI the threshold analysis for comb generation. The role of cavity dispersion is specifically investigated in Sec. VII, while Sec. VIII is dedicated to the study of the comb dynamics above threshold. We discuss the various possibilities to expand the comb span beyond one octave in Sec. IX, and the last section concludes the article.

\section{THE SYSTEM UNDER STUDY}

The typical scheme of a WGM comb generator is displayed in Fig. 1. A continuous-wave laser with a very narrow linewidth is used to pump a nonlinear dielectric cavity. After polarization control, the laser beam is coupled into a resonant cavity mode using evanescent fields. The intracavity photons interact

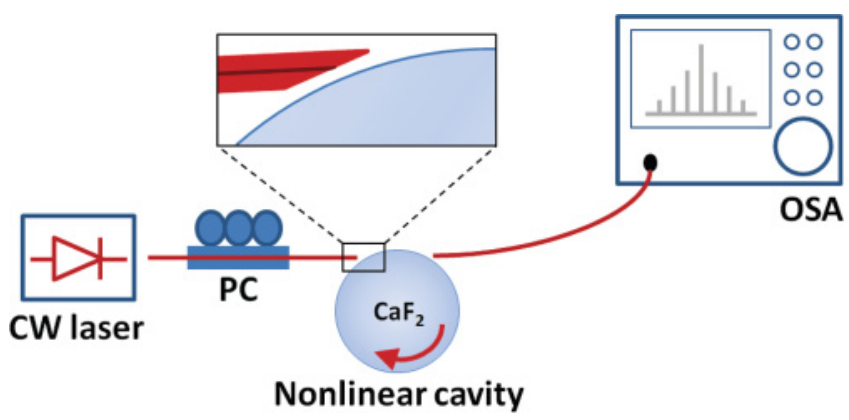

FIG. 1. (Color online) Optical-frequency comb generator with a calcium fluoride cavity and angle-polished fiber couplings. CW, continuous-wave; PC, polarization control; OSA, optical spectrum analyzer. The OSA displays the spectrum of the optical-frequency comb. through FWM and generate the optical-frequency comb, whose evanescent field can also be extracted and monitored with an optical spectrum analyzer.

Optical-frequency comb generators can be made of a wide variety of WGM resonators. The first element to consider is the size of the cavity (characterized by its circumference), which determines the FSR of the comb. The bulk medium filling the cavity can be amorphous (e.g., fused silica) or crystalline (e.g., calcium fluoride). In the latter case, however, an inversion symmetry is required for the Kerr nonlinearity to be significant. The evanescent coupling is generally performed using prisms or tapered or angle-polished fibers. So far, comb generation has been observed with cavities whose $Q$ factors range from $10^{7}$ to $10^{10}$. The bulk cavity is generally a toroid or a disk whose rim has a local curvature (truncated sphere or ovoid).

We develop the theoretical framework independently of the various elements cited earlier. In particular, we have built a theory that is valid for optical-frequency combs generated with any cavity with axial symmetry, provided that the bulk medium is homogeneous and isotropic. In this study, we focus without loss of generality on spherical $\mathrm{CaF}_{2}$ resonators with FSR of about $10 \mathrm{GHz}$ and optical wavelength at $1560 \mathrm{~nm}$.

\section{THE MULTIMODE APPROACH TO COMB GENERATION IN WGM RESONATORS}

Modeling a spatiotemporal system typically corresponds to establish a (set of) partial differential equation(s) describing what is occurring in each point and at each instant within the volume of interest. Even though this full spatiotemporal description is synthetic and elegant, it has some severe limitations. A typical case is when the system is sharply multimodal. In that case, if the spatial distribution of the modes can be analytically determined, the spatiotemporal modeling is redundant as only the temporal variation of the modal amplitudes is unknown. Moreover, the redundant part is the most difficult part of theoretical analysis (partial differential equations) and the most time-consuming part of an eventual numerical modeling (finite element algorithms).

Modal expansion methods, on the other hand, rely on the fact that when spatial mode distributions are known (this is an essential point), the initial spatiotemporal equation can be decomposed into a finite set of coupled time-domain differential equations for the modal amplitudes. These models outperform spatiotemporal models in this context by speeding up numerical simulations and decreasing complexity; in particular, the dimensionality of the governing equations drops sharply from infinity to a finite value, of the order of the number of modes. Modal expansion also enables simpler stability analysis and analytical treatment, and it allows an easier determination of some critical parameters such as modal characteristics (linewidths, frequency shifts, coupling, etc.), oscillation thresholds, and bifurcation values.

The starting point of our analysis will therefore be the Maxwell wave equation in a bulk spherical cavity that is assumed to be absorptive, dispersive, and nonlinear. We consider an homogeneous and isotropic dielectric sphere of radius $a$, submitted to an external electromagnetic field of frequency $\Omega_{0}$ (in our case, the laser excitation). The electric field therefore obeys everywhere (inside and outside the cavity) 
the classical wave equation

$$
\left[\Delta-\frac{\epsilon\left(\mathbf{r}, \omega,\|\mathbf{E}\|^{2}\right)}{c^{2}} \frac{\partial^{2}}{\partial t^{2}}\right] \mathbf{E}(\mathbf{r}, t)=\mathbf{0},
$$

where the relative permittivity $\epsilon$ is defined as

$$
\epsilon\left(\mathbf{r}, \omega,\|\mathbf{E}\|^{2}\right)= \begin{cases}n^{2}\left(\omega,\|\mathbf{E}\|^{2}\right) & \text { if } r \leqslant a \\ 1 & \text { if } r>a\end{cases}
$$

with $n$ being the refractive index of the medium. Note that we can legitimately keep the frequency dependence of $\epsilon$ in the spatiotemporal Eq. (1) because this dependence is very weak relative to the Fourier transform of the electric field and its derivatives.

Since we know a priori that the cavity is sharply resonant around its eigenmodes, we can expand this field as

$\mathbf{E}(\mathbf{r}, t)=\sum_{\mu} \frac{1}{2} \mathcal{E}_{\mu}(t) e^{i \omega_{\mu} t} \Upsilon_{\mu}(\mathbf{r})+\frac{1}{2} \mathcal{E}_{\text {ext }} e^{i \Omega_{0} t} \mathbf{e}_{0}+$ c.c.,

where $\mu$ labels the various modes under consideration, defined by an infinite set of orthonormal and vectorial eigenmodes $\Upsilon_{\mu}(\mathbf{r})$ of absolute frequency $\omega_{\mu}$ and by their time-varying amplitude $\mathcal{E}_{\mu}(t)$. The direction of the external pumping field $\mathcal{E}_{\text {ext }}$ is defined by the unit vector $\mathbf{e}_{0}$ whose modulus has the dimension of a volumic density, while c.c. stands for the complex conjugate of all the preceding terms. This expansion, sometimes referred to as a slowly varying amplitude (SVA) expansion in the literature, implicitly assumes the spatial and temporal variations of the field are separable; hence propagation effects are not accounted for, as a mode is assumed to have the same amplitude everywhere. This amplitude of the field is also generally assumed to vary slowly relative to its nominal frequency [i.e., $\left|\dot{\mathcal{E}}_{\mu}(t)\right| \ll \omega_{\mu}\left|\mathcal{E}_{\mu}(t)\right|$ ]. Note that here we consider that the external field is nearly resonant with the cavity, in the sense that $\Omega_{0}$ is near an eigenfrequency of the system. It is a free parameter, tunable within a few bandwidths around the resonance frequency of the excited mode. This pump frequency will also be a reference for all practical purposes and in particular for the refractive index as we consider $n_{0} \equiv \operatorname{Re}\left[n\left(\Omega_{0}\right)\right]$.

The parameter that determines all the dynamical features of this system is the refractive index $n\left(\omega,\|\mathbf{E}\|^{2}\right)$. It contains all the information about the dielectric material under study. The three key phenomena we need to consider here are the linear absorption, relevant for the $Q$ factor of the dielectric cavity; the chromatic dispersion, which discriminates the various optical frequencies in terms of refractive index; and the Kerr nonlinearity, responsible for four-wave mixing. Therefore we have to define how these three features should be introduced in our dielectric parameter.

At this semiclassical level, linear absorption is generally introduced as a small imaginary part in the refractive index so that the refraction index becomes $n_{0}-i n_{a}(\omega)$, with $n_{a}(\omega)>0$ for all $\omega$ (no lasing effects). Loss of energy in this cavity is because of factors that are both internal (material absorption, radiative losses, volume or surface scattering, etc.) and external (out-coupling process) so that we can write $n_{a}(\omega)=n_{\text {int }}(\omega)+$ $n_{\text {ext }}(\omega)$. The frequency dependence of material absorption is negligible around the pump frequency but not for relatively wide frequency spans. On the other hand, out-coupling losses are more frequency dependent for neighboring frequencies, and the particular features of this frequency dependence are defined by the coupling method. It is, however, important to note that the out-coupling losses can be experimentally tuned. This degree of freedom will be exploited further in the article. Dispersion can be accounted for by allowing a frequencydependent component to the real part of the refraction index around the central frequency, following $n(\omega)=n_{0}+n_{d}(\omega)$, with $n_{d}\left(\Omega_{0}\right)=0$. This dispersion term is relevant even very near the pump frequency. Finally, Kerr nonlinearity modifies the refraction index following $n_{0}+n_{2} I$, where $n_{2}$ is the nonlinear Kerr factor and $I$ is the optical intensity (irradiance) of the field.

These three corrections are small relative to the real refractive index $n_{0}$. Therefore, in the medium, the relative permittivity can be explicitly expressed as

$$
\begin{aligned}
\epsilon\left[r \leqslant a, \omega,\|\mathbf{E}\|^{2}\right] & =\left[n(\omega)+\Delta n\left(\omega,\|\mathbf{E}\|^{2}\right)\right]^{2} \\
& \simeq n^{2}(\omega)+2 n_{0} \Delta n\left(\omega,\|\mathbf{E}\|^{2}\right),
\end{aligned}
$$

where $n(\omega)$ is real and includes the dispersive characteristics of the refraction index, while

$$
\Delta n\left(\omega,\|\mathbf{E}\|^{2}\right)=-i\left[n_{\mathrm{int}}(\omega)+n_{\mathrm{ext}}(\omega)\right]+n_{2} \frac{n_{0} \varepsilon_{0} c}{2}\|\mathbf{E}\|^{2}
$$

stands for the complex and nonlinear corrections to $n_{0}, \varepsilon_{0}$ being the vacuum permittivity.

Using Eqs. (3) and (4), we can finally rewrite the initial Eq. (1) under the form

$$
\begin{aligned}
& \sum_{\mu} \mathcal{E}_{\mu}(t) e^{i \omega_{\mu} t}\left\{\Delta+\frac{\omega_{\mu}^{2}}{c^{2}} \operatorname{Re}[\epsilon(\mathbf{r}, \omega, 0)]\right\} \boldsymbol{\Upsilon}_{\mu}(\mathbf{r}) \\
& +\sum_{\mu}\left\{2 n_{0} \Delta n\left(\omega,\|\mathbf{E}\|^{2}\right) \frac{\omega_{\mu}^{2}}{c^{2}} \mathcal{E}_{\mu}(t)\right. \\
& \left.-\frac{\epsilon\left(\mathbf{r}, \omega,\|\mathbf{E}\|^{2}\right)}{c^{2}}\left[\ddot{\mathcal{E}}_{\mu}(t)+2 i \omega_{\mu} \dot{\mathcal{E}}_{\mu}(t)\right]\right\} e^{i \omega_{\mu} t} \boldsymbol{\Upsilon}_{\mu}(\mathbf{r}) \\
& +\frac{\epsilon\left(\mathbf{r}, \omega,\|\mathbf{E}\|^{2}\right)}{c^{2}} \Omega_{0}^{2} \mathcal{E}_{\mathrm{ext}} e^{i \Omega_{0} t} \mathbf{e}_{0}=\mathbf{0}
\end{aligned}
$$

This equation can be separated into two distinct parts. The first part (spatial) is the first term. It should be equated to zero as $\Upsilon_{\mu}(\mathbf{r})$ is by definition an eigenmode. The resolution of the eigenmode equation will then give us an explicit solution for $\Upsilon_{\mu}(\mathbf{r})$, which will be injected in the second part of the equation (temporal), which is constituted with the remaining terms of Eq. (6). This procedure will later enable us to obtain time-domain ordinary differential equations for the modal amplitudes $\mathcal{E}_{\mu}(t)$.

\section{ANALYTICAL DETERMINATION OF THE WGMS}

\section{A. Eigenmode solutions}

The eigenmodes are solutions of the equation

$$
\left[\Delta+\frac{\omega_{\mu}^{2}}{c^{2}} \epsilon\left(r, \omega_{\mu}\right)\right] \Upsilon_{\mu}(\mathbf{r})=\mathbf{0},
$$

with $\epsilon(r, \omega)=n^{2}(\omega)$ for $r \leqslant a$ and $\epsilon(r, \omega)=1$ for $r>a$. 
In order to solve this problem, a convenient method is to first set $n(\omega)$ to $n_{0}$, that is, to neglect dispersion. This equation can then be solved exactly in spherical coordinates, and the solutions can either be transverse electric (TE) or transverse magnetic (TM), following [20]

$$
\begin{gathered}
\boldsymbol{\Upsilon}_{\ell m n}^{\mathbf{T E}}(\mathbf{r})=\frac{e^{i m \phi}}{k_{\ell n p} r} S_{\ell n p}(r) \mathbf{X}_{\ell m}(\theta) \\
\Upsilon_{\ell m n}^{\mathbf{T M}}(\mathbf{r})=\frac{e^{i m \phi}}{k_{\ell n p}^{2} n_{0}^{2}}\left\{\frac{1}{r} \frac{d}{d r} S_{\ell n p}(r) \mathbf{Y}_{\ell m}(\theta)+\frac{1}{r^{2}} S_{\ell n p}(r) \mathbf{Z}_{\ell m}(\theta)\right\}
\end{gathered}
$$

where $p$ stands for TE in the first equation and for TM in the second. Also note that here $\theta$ is the colatitude while $\phi$ is the longitude. The spherical vectors are explicitly defined as

$$
\begin{gathered}
\mathbf{X}_{\ell m}(\theta)=i \frac{m}{\sin \theta} P_{\ell}^{m}(\cos \theta) \mathbf{e}_{\theta}-\frac{\partial}{\partial \theta} P_{\ell}^{m}(\cos \theta) \mathbf{e}_{\phi}, \\
\mathbf{Y}_{\ell m}(\theta)=\frac{\partial}{\partial \theta} P_{\ell}^{m}(\cos \theta) \mathbf{e}_{\theta}-i \frac{m}{\sin \theta} P_{\ell}^{m}(\cos \theta) \mathbf{e}_{\phi}, \\
\mathbf{Z}_{\ell m}(\theta)=\ell(\ell+1) P_{\ell}^{m}(\cos \theta) \mathbf{e}_{r},
\end{gathered}
$$

where $P_{\ell}^{m}(\cos \theta)$ are the associated Legendre polynomials, $m$ and $\ell$ being two integers fulfilling $-\ell \leqslant m \leqslant \ell$.

The function $S_{\ell n p}$ is the radial Debye potential

$$
S_{\ell n p}(r)= \begin{cases}\psi_{\ell}\left(n_{0} k_{\ell n p} r\right) & \text { if } r \leqslant a, \\ \frac{\psi_{\ell}\left(n_{0} k_{\ell n p} a\right)}{\chi_{\ell}\left(k_{\ell n p} a\right)} \chi_{\ell}\left(k_{\ell n p} r\right) & \text { if } r>a,\end{cases}
$$

where $k_{\ell n p}$ is the $n$th solution of the algebraic equation

$$
\frac{\chi_{\ell}^{\prime}(k a)}{\chi_{\ell}(k a)}=p \frac{\psi_{\ell}^{\prime}(k a)}{\psi_{\ell}(k a)} \quad \text { with } \quad p=\left\{\begin{array}{lll}
n_{0} & \text { for } & \text { TE modes, } \\
1 / n_{0} & \text { for } & \text { TM modes }
\end{array}\right.
$$

while $\psi_{\ell}$ and $\chi_{\ell}$ are the $\ell$ th-order Riccati-Bessel functions.

\section{B. THE WGMS}

WGMs are generally defined as those cavity modes for which the electric field is strongly confined in a narrow torus near the equatorial circle and is quasinull everywhere else. Mathematically, this configuration corresponds to the fundamental radial mode with large but equal polar and azimuthal eigenvalues (i.e., $n=1$ and $m \equiv \ell \gg 1$ ). In that case, having in mind that

$$
P_{\ell}^{\ell}(\cos \theta)=\left[(-1)^{\ell}(2 \ell-1) ! !\right] \sin ^{\ell} \theta
$$

and $\theta \simeq \pi / 2$, it can be easily shown from Eqs. (10) that the TE mode becomes asymptotically parallel to $\mathbf{e}_{\theta}$, while the TM mode becomes parallel to $\mathbf{e}_{r}$, as both $\partial_{\theta} P_{\ell}^{\ell}(\cos \theta)$ and $\left\|\mathbf{Y}_{\ell \ell}(\theta)\right\| /\left\|\mathbf{Z}_{\ell \ell}(\theta)\right\|$ do vanish when $\theta \rightarrow \pi / 2$. Hence our eigenvectors only depend on two parameters: the angular eigenvalue $\ell$, which is a degenerated scalar parameter, and the vectorial polarization $p$, standing for TE or TM modes.

It has also been shown earlier in the literature that for this resonator, the eigenfrequencies can be approximated as [21]

$$
\begin{aligned}
\omega_{\ell p}= & k_{\ell 1 p} c \\
= & \frac{c}{n\left(\omega_{\ell p}\right) a}\left\{\left[\ell+\frac{1}{2}\right]+\xi_{1}\left[\frac{\ell+\frac{1}{2}}{2}\right]^{1 / 3}-\frac{p}{\sqrt{n_{0}^{2}-1}}\right. \\
& \left.+\frac{3}{20} \xi_{1}^{2}\left[\frac{\ell+\frac{1}{2}}{2}\right]^{-1 / 3}+O\left(\ell+\frac{1}{2}\right)^{-2 / 3}\right\}
\end{aligned}
$$

where $p$ is polarization dependent, as explicitly defined in Eq. (12), while $\xi_{n}$ is the $n$th root of the Airy function $\mathrm{Ai}(-z)$ and corresponds to the $n$th radial order. For WGMs, we have $n=1$ and $\xi_{1}=2.338$. It is noteworthy that these eigenfrequencies are weakly unequidistant because of material and geometrical dispersion. Note that material dispersion has been introduced a posteriori as a perturbation of the solutions of the dispersionless cavity.

As a specific example, we use a resonator of principal radius $a=2.5 \mathrm{~mm}$, with $n_{0}=1.43$ at the central wavelength $\lambda_{0}=1560.5 \mathrm{~nm}$ (in vacuum). In the vicinity of the pump, the angular number can be roughly estimated as $\ell_{0} \sim 2 \pi a n_{0} / \lambda_{0} \sim 14400$, and the free-spectral range (FSR) can also be simply evaluated as

$$
\Delta \omega_{\mathrm{FSR}} \simeq \frac{c}{a n_{0}} \simeq 2 \pi \times 13.36 \mathrm{GHz} .
$$

It is interesting to note that this FSR corresponds to the period of the photons inner circumferential motion (velocity $c / n_{0}$ divided by the circumference $2 \pi a$ ).

\section{Normalization}

It is convenient to normalize the eigenvectors in this analytical treatment because it simplifies calculations, and it also enables us to have direct access to quantitative values. Some important approximations, very useful for the numerical computation of the modes, will also be outlined.

The orthonormal solutions of Eq. (7) that we are going to use in this article can therefore be labeled as $\Upsilon_{\ell p}(\mathbf{r})$, and they are such that

$$
\boldsymbol{\Upsilon}_{\ell, T E}(\mathbf{r})=\frac{\Upsilon_{\ell \ell 1}^{\mathrm{TE}}}{N_{\ell, T E}}, \quad \Upsilon_{\ell, T M}(\mathbf{r})=\frac{\Upsilon_{\ell \ell 1}^{\mathrm{TM}}}{N_{\ell, T M}}
$$

where the normalization constants $N_{\ell p}$ are obtained through the orthonormalization condition

$$
\int_{\infty} \Upsilon_{\ell p}^{*}(\mathbf{r}) \cdot \Upsilon_{\ell^{\prime} p^{\prime}}(\mathbf{r}) d V=\delta_{\ell \ell^{\prime}} \delta_{p p^{\prime}}
$$

While carrying out the integrals of Eq. (17), it should be recalled that the WGMs are located near the equator at $\theta \simeq \theta_{0}=\pi / 2$ so that at the first order, we have $\sin \theta \sim 1$ and $\cos \theta \simeq \theta_{0}-\theta \rightarrow 0$. Moreover, it is known that spherical modes degenerate to Gaussian modes when $\ell=m$ (see Ref. [8]), and we have

$$
P_{\ell}^{\ell}(x) \simeq P_{\ell}^{\ell}(0) e^{-\frac{1}{2} \ell x^{2}}
$$


so that the polar integral can be simplified as

$$
\begin{aligned}
\int_{0}^{\pi}\left[P_{\ell}^{\ell}(\cos \theta)\right]^{2} \sin \theta d \theta & \simeq\left[P_{\ell}^{\ell}(0)\right]^{2} \int_{-\infty}^{+\infty} e^{-\ell\left(\theta-\theta_{0}\right)^{2}} d \theta \\
& =[(2 \ell-1) ! !]^{2} \sqrt{\frac{\pi}{\ell}}
\end{aligned}
$$

Here we have considered that the poles are at infinity relative to the equator for the WGMs, whose polar dependence is Gaussian with a typical waist of the order of $1 / \sqrt{\ell}$ $\sim 10^{-2} \mathrm{rad}$. The radial integral can also be simplified as we know that the electric field is quasinull everywhere except in a narrow torus near the circumference, whose tubular radius is only few wavelengths wide; hence the variable $r$ can be replaced by $a$ whenever it is not an argument of the Debye potential $S_{\ell 1 p}$, and we can write

$$
\int_{0}^{+\infty} S_{\ell 1 p}^{2}(r) r^{2} d r \simeq a^{2} \int_{\sim a} S_{\ell 1 p}^{2}(r) d r,
$$

where $\sim a$ stands for the neighborhood of $a$, equivalent here for all practical purposes to a span of few tens of $\lambda$ around $a$. This approximation is extremely useful as it significantly speeds up (at least by a factor 100) the computation time of the modes and related parameters with almost no penalty in accuracy. However, in the case of microresonators, the ratio $\lambda / a$ is not so small, and one may prevent a loss of accuracy by keeping the $r^{2}$ term inside the integral, still integrating over the neighborhood of $a$.

Finally, the normalized eigenvectors $\Upsilon_{\ell p}(\mathbf{r})$ explicitly read

$$
\begin{gathered}
\Upsilon_{\ell, \mathrm{TE}}(\mathbf{r})=i \Upsilon_{\ell, \mathrm{TE}}(r, \theta, \phi) \mathbf{e}_{\theta} \\
\Upsilon_{\ell, \mathrm{TM}}(\mathbf{r})=\Upsilon_{\ell, \mathrm{TM}}(r, \theta, \phi) \mathbf{e}_{r}
\end{gathered}
$$

with

$$
\Upsilon_{\ell p}(r, \theta, \phi)=\frac{(-1)^{\ell} \ell^{\frac{1}{4}}}{2^{\frac{1}{2}} \pi^{\frac{3}{4}} a} \frac{S_{\ell 1 p}(r) e^{-\frac{1}{2} \ell\left(\theta-\frac{\pi}{2}\right)^{2}} e^{i \ell \phi}}{\sqrt{\int_{\sim a} S_{\ell 1 p}^{2}(r) d r}} .
$$

Figure 2 displays the color-coded two-dimensional variations of a WGM cross section, according to Eq. (22). It can be seen that it consists of a strongly confined bright spot whose transverse spatial dimension is of the order of a few

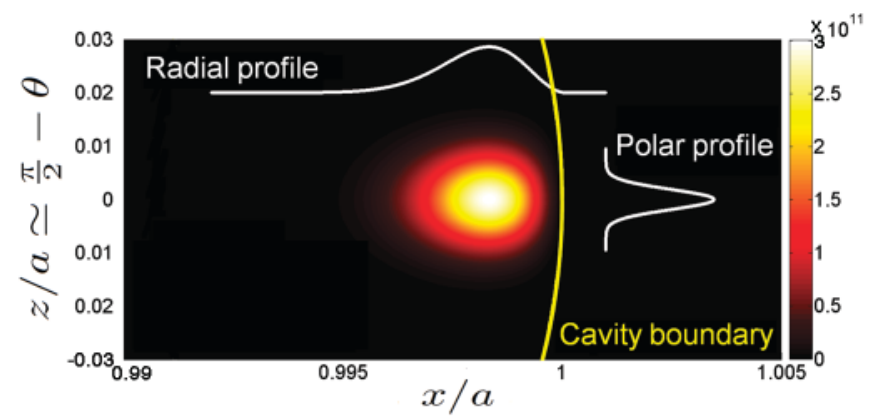

FIG. 2. (Color online) Cross section at $\phi=0$ (half-plane $y=0$ and $x>0)$ of the whispering gallery mode $\left\|\boldsymbol{\Upsilon}_{\ell, T E}(\mathbf{r})\right\|^{2}$ in units of $\mathrm{m}^{-3}$. The value of the polar eigennumber is $\ell_{0}=14350$, corresponding to $\lambda_{0}=1560.5 \mathrm{~nm}$ in vacuum. The spherical $\mathrm{CaF}_{2}$ cavity has a radius $a=2.5 \mathrm{~mm}$ and a refraction index $n_{0}=1.43$ at $\lambda_{0}$. Note that the axes are not orthonormal: the radial confinement is in fact 10 times stronger than the polar one.
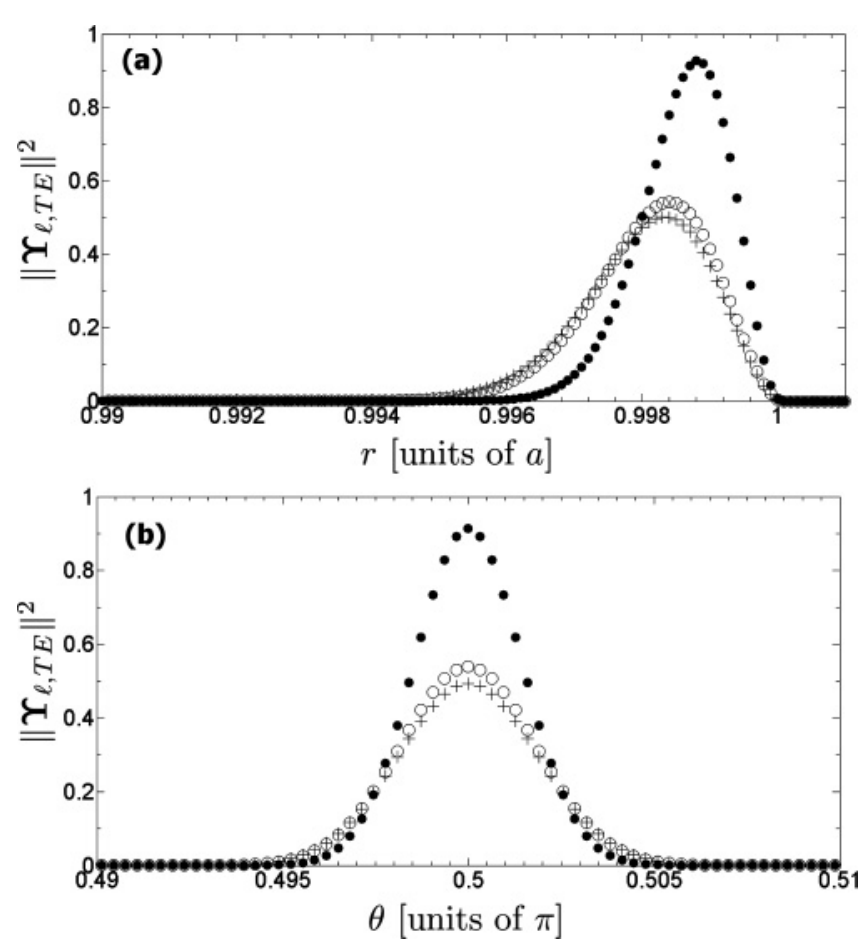

FIG. 3. Radial and polar profiles of the modes (arbitrary units) for $\ell_{0}=14350$ (plusses), for $\ell_{0}+1000$ (open circles), and for $\ell_{0}+10000$ (solid circles). (a) Radial profiles for $\theta=0$ and $\phi=0$. (b) Polar profiles for $\phi=0$ at intensity maxima. All other parameters are those of Fig. 2.

wavelengths. For the values considered in this figure, the confinement is in fact much stronger in the radial dimension (typical waist of $\sim 0.001 a)$ than in the polar one $(\sim 0.01 a)$. This issue of relatively poor polar confinement can be solved using toroidal cavities whose local curvature is smaller and thereby does not allow for a large polar spreading [4]. Another alternative is to use a disk whose rim radius is significantly smaller than the principal radius [6,7] (this geometry somehow corresponds to a toroidal cavity as well).

Figure 3 focuses on how the WGM is modified as the eigennumber $\ell_{0}$ is changed. Figure 3(a) shows that when $\ell_{0}$ increases, the mode becomes more radially confined, while its maximum becomes pronounced (increasing as $\sqrt{\ell}$ ). On the other hand, Fig. 3(b) also indicates that increasing the polar eigennumber enhances the polar confinement, with a waist decreasing as $1 / \sqrt{\ell}$. Both figures evidence that even WGMs separated by 1000 modes (a spectral span of $\sim 100 \mathrm{~nm}$ in our case) are still quite satisfyingly overlapped and therefore may experience strong mutual coupling. However, if the spectral gap increases too much, for example, to 10000 free spectral ranges, then the spatial overlap is severely deteriorated, and the modes would be poorly coupled.

\section{THE MODAL EXPANSION MODEL}

As explained earlier, the first term of Eq. (6) vanishes, and we have found that $\mu=\{\ell, p\}$; that is, a WGM is exclusively characterized by its angular number and its polarization. This information should be used to derive the temporal behavior of the modes from the remaining terms. 
Additional useful approximations can be introduced in these remaining terms. Owing to the slowly varying amplitude assumption, we can consider that $\left|\ddot{\mathcal{E}}_{\mu}(t)\right| \ll\left|2 \omega_{\mu} \dot{\mathcal{E}}_{\mu}(t)\right|$ so that the second derivative term can be neglected. Moreover, the relative permittivity $\epsilon\left(\mathbf{r}, \omega,\|\mathbf{E}\|^{2}\right)$ can now be set to the reference value $\epsilon\left(\mathbf{r}, \Omega_{0}, 0\right)$ as the perturbation $\Delta n$ can be neglected at this stage when standing beside the principal value $n_{0}$. Therefore Eq. (6) can be rewritten as

$$
\begin{aligned}
\sum_{\mu} & \omega_{\mu} \dot{\mathcal{E}}_{\mu}(t) e^{i \omega_{\mu} t} \boldsymbol{\Upsilon}_{\mu}(\mathbf{r}) \\
= & \sum_{\mu}-i \omega_{\mu}^{2} \frac{n_{0} \Delta n\left(\omega,\|\mathbf{E}\|^{2}\right)}{\epsilon\left(\mathbf{r}, \Omega_{0}, 0\right)} \mathcal{E}_{\mu}(t) e^{i \omega_{\mu} t} \boldsymbol{\Upsilon}_{\mu}(\mathbf{r}) \\
& -\frac{1}{2} i \Omega_{0}^{2} \mathcal{E}_{\mathrm{ext}} e^{i \Omega_{0} t} \mathbf{e}_{0} .
\end{aligned}
$$

The preceding equation is a single global equation ruling the collective dynamics of the modes, whereas we are interested in the individual dynamics. However, owing to the orthonormality of the eigenvectors, we can target the individual dynamics $\mathcal{E}_{\eta}(t)$ of a given mode $\eta$ by projecting this global equation onto $\Upsilon_{\eta}(\mathbf{r})$, in other words, by multiplying Eq. (23) by $\Upsilon_{\eta}^{*}(\mathbf{r}) e^{-i \omega_{\eta} t}$ and spatially integrating over infinity (Hermitian inner product). This projection gives the following result:

$$
\begin{aligned}
\dot{\mathcal{E}}_{\eta}= & -\frac{1}{\omega_{\eta}} \int_{V} \sum_{\mu} i \omega_{\mu}^{2} \frac{\Delta n\left(\omega,\|\mathbf{E}\|^{2}\right)}{n_{0}} \mathcal{E}_{\mu} e^{i\left(\omega_{\mu}-\omega_{\eta}\right) t} \\
& \times\left[\Upsilon_{\eta}^{*}(\mathbf{r}) \cdot \Upsilon_{\mu}(\mathbf{r})\right] d V \\
& -\frac{1}{\omega_{\eta}} \int_{\infty} \frac{1}{2} i \Omega_{0}^{2} \mathcal{E}_{\mathrm{ext}} e^{i\left(\Omega_{0}-\omega_{\eta}\right) t}\left[\Upsilon_{\eta}^{*}(\mathbf{r}) \cdot \mathbf{e}_{0}\right] d V
\end{aligned}
$$

Note that the first integral is only over the dielectric volume $V$ because $\Delta n \equiv 0$ outside the cavity.

It may also be convenient to normalize the electric field as

$$
\mathcal{A}_{\eta}=\sqrt{\frac{1}{2} \frac{\varepsilon_{0} n_{0}^{2}}{\hbar \omega_{\eta}}} \mathcal{E}_{\eta},
$$

where $\left|\mathcal{A}_{\eta}\right|^{2}$ is the instantaneous number of photons in the mode $\eta$ (they are mostly inside the cavity). We can use Eq. (5) and

$$
\|\mathbf{E}\|^{2}=\sum_{\alpha, \beta} \mathcal{E}_{\alpha} \mathcal{E}_{\beta}^{*} e^{i\left(\omega_{\alpha}-\omega_{\beta}\right) t}\left[\Upsilon_{\beta}^{*}(\mathbf{r}) \cdot \Upsilon_{\alpha}(\mathbf{r})\right]
$$

to obtain finally the following explicit rate equations for the modal field dynamics:

$$
\begin{aligned}
\dot{\mathcal{A}}_{\eta}= & -\frac{1}{2} \Delta \omega_{\eta} \mathcal{A}_{\eta}-i g_{0} \sum_{\alpha, \beta, \mu} \Lambda_{\eta}^{\alpha \beta \mu} \mathcal{A}_{\alpha} \mathcal{A}_{\beta}^{*} \mathcal{A}_{\mu} e^{i \varpi_{\alpha \beta \mu \eta} t} \\
& +\frac{1}{2} \Delta \omega_{\eta} \mathcal{F}_{\eta} e^{i\left(\Omega_{0}-\omega_{\eta}\right) t},
\end{aligned}
$$

which enable us to track the field amplitude in each mode $\eta$. The parameters of Eq. (27) are defined and discussed hereinafter.

The modal bandwidth

$$
\begin{aligned}
\Delta \omega_{\eta} & =2 \Gamma_{\eta} \frac{\omega_{\eta}}{n_{0}}\left[n_{\mathrm{int}}\left(\omega_{\eta}\right)+n_{\mathrm{ext}}\left(\omega_{\eta}\right)\right] \\
& =\Delta \omega_{\mathrm{int}, \eta}+\Delta \omega_{\mathrm{ext}, \eta}
\end{aligned}
$$

obtained through this modal expansion formalism is consistent with results found in the literature [20,22,23], and it is physically the inverse of the modal photon lifetime $\tau_{\eta}$. We find that the modal linewidth linearly depends on its central frequency $\omega_{\eta}$ and on the loss coefficients $n_{\text {int }}$ and $n_{\text {ext }}$, which are themselves frequency dependent. The material loss contribution to the modal bandwidth is fixed once and for all and exclusively depends on the laser pumping frequency. It is interesting to note that the absorption of $\mathrm{CaF}_{2}$ can vary significantly over large wavelength spans. On the other hand, the coupling-dependent contribution $\Delta \omega_{\text {ext }}$ strongly depends on the coupling architecture (prism, angle-polished or tapered fiber, etc.), and the three main configurations are undercoupling $\left(\Delta \omega_{\text {ext }}<\Delta \omega_{\text {int }}\right)$, critical coupling $\left(\Delta \omega_{\text {ext }}=\right.$ $\left.\Delta \omega_{\text {int }}\right)$, and overcoupling $\left(\Delta \omega_{\text {ext }}>\Delta \omega_{\text {int }}\right)$ [24-26]. The modal bandwidth is also proportional to the confinement factor

$$
\Gamma_{\eta}=\int_{V}\left\|\boldsymbol{\Upsilon}_{\eta}(\mathbf{r})\right\|^{2} d V
$$

measuring the energy portion of the mode that is within the sphere. It should be noted that even though all the WGMs have the same radial order $n=1$, they have different angular orders $\ell$ and therefore different radial profiles according to Eq. (11). Since the WGMs are strongly confined, this factor is very close to 1 . Moreover, modes with close order $\ell$ also have quasiequal confinement factors, however, this is not the case for modes that are spectrally far away.

The four-wave mixing reference gain can be defined as

$$
g_{0}=\frac{n_{2} c}{n_{0}^{2}} \frac{\hbar \omega_{\eta_{0}}^{2}}{V_{\eta_{0}}}
$$

where $\omega_{\eta_{0}}$ is the eigenfrequency of the pumped mode $\eta_{0}$, while $V_{\eta_{0}}$ is its effective volume:

$$
V_{\eta}=\left[\int_{V}\left\|\mathbf{\Upsilon}_{\eta}(\mathbf{r})\right\|^{4} d V\right]^{-1} .
$$

This corresponds to the nonlinear gain for the modes that are in the immediate neighborhood of the pump frequency $\Omega_{0} \simeq \omega_{\eta_{0}}$. It immediately follows that it is proportional to the Kerr nonlinearity but inversely proportional to the effective mode volume.

The intermodal coupling factor

$$
\Lambda_{\eta}^{\alpha \beta \mu}=\frac{\omega_{\mu}^{2}}{\omega_{\eta_{0}}^{2}} \sqrt{\frac{\omega_{\alpha} \omega_{\beta} \omega_{\mu}}{\omega_{\eta}^{3}}} \frac{\int_{V}\left[\mathbf{\Upsilon}_{\eta}^{*} \cdot \boldsymbol{\Upsilon}_{\mu}\right]\left[\mathbf{\Upsilon}_{\beta}^{*} \cdot \boldsymbol{\Upsilon}_{\alpha}\right] d V}{\int_{V}\left\|\boldsymbol{\Upsilon}_{\eta_{0}}\right\|^{4} d V}
$$

defines the coupling strength between the four interacting modes $\alpha, \beta, \mu$, and $\eta$. This coupling essentially depends on the power density overlap of the modes. The modes of a narrow-span comb (close-by values of $\ell$ ) have approximately degenerated values for this coupling factor. Modes that are far away can be relatively poorly coupled as their power density overlap is not optimal (see Fig. 3). Hence the pumped WGM is geometrically strongly coupled to its adjacent modes but less efficiently coupled to extreme modes. This is another factor that should be considered while analyzing the spanlimiting mechanisms in optical-frequency comb generation. Interestingly, according to Eq. (32), this term does not vanish when we consider pairwise crossed-polarized photons (e.g., $\alpha$ and $\beta$ are TE modes while $\mu$ and $\eta$ are TM modes). 
The ideal resonance condition occurs when the modal fourwave mixing frequency detuning

$$
\varpi_{\alpha \beta \mu \eta}=\omega_{\alpha}-\omega_{\beta}+\omega_{\mu}-\omega_{\eta}
$$

vanishes. This condition straightforwardly imposes that any four photons involved in the four-wave mixing interactions $\hbar \omega_{\alpha}+\hbar \omega_{\mu} \rightarrow \hbar \omega_{\beta}+\hbar \omega_{\eta}$ should obey energy conservation as well as the conservation of total angular momentum following $\ell_{\alpha}+\ell_{\mu}=\ell_{\beta}+\ell_{\eta}$. This ideal condition $\varpi_{\alpha \beta \mu \eta}=0$ would have been automatically fulfilled for an equidistant eigenmode distribution, but since there is no such equidistance in WGM resonators, $\varpi_{\alpha \beta \mu \eta}$ walks off from 0 . In fact, four-wave mixing is a priori still possible for a mode $\eta$ as long as the walk-off is smaller than the modal half-bandwidth, that is, $\left|\varpi_{\alpha \beta \mu \eta}\right|<\Delta \omega_{\eta} / 2$. In reality, other detunings, such as those induced by self- or cross-modulation, can impede the phenomenon. Generally, in the literature, this walk-off from perfect equidistance is considered to be the main mechanism leading to a limited span for the comb. However, as we have seen in the preceding paragraphs, many other phenomena should be taken into account while investigating the spanlimiting mechanisms (nonuniform absorption, nondegenerate confinement factors, power overlap coupling for the modes, etc.).

Finally, the external pumping term

$$
\mathcal{F}_{\eta}=-\frac{i \Omega_{0}^{2}}{\omega_{\eta} \Delta \omega_{\eta}} \sqrt{\frac{1}{2} \frac{\varepsilon_{0} n_{0}^{2}}{\hbar \omega_{\eta}}} \mathcal{E}_{\text {ext }} \int_{\infty} \mathbf{\Upsilon}_{\eta}^{*}(\mathbf{r}) \cdot \mathbf{e}_{0} d V
$$

quantifies how the external pumping interacts with the WGM $\eta$. This term is only resonant with the reference mode of frequency $\omega_{\eta_{0}} \simeq \Omega_{0}$ and is totally rejected for any other mode (since, in that case, $\left|\Omega_{0}-\omega_{\eta}\right| \gg \Delta \omega_{\eta}$ ). It has been normalized in a way that $\mathcal{F}_{\eta}$ has a comparable magnitude with $\mathcal{A}_{\eta}$; in particular, $\left|\mathcal{F}_{\eta_{0}}\right|^{2}$ can be interpreted as the number of photons that are coupled into the cavity from the outside. On the basis of this modal expansion model, we will investigate in detail the threshold mechanisms of comb generation in the next section.

\section{COMB-GENERATION THRESHOLD}

For the sake of simplicity, we only consider in this article the simplest configuration, where the pumping field has a single frequency and a fixed polarization. Therefore, starting from this section, we assume that the modes $\eta$ only depend on their polar number $\ell$. For convenience, we introduce a shifted eigennumber $l=\ell-\ell_{0}$, where $\ell_{0}$ is the angular number of the pumped mode. This notation is particularly interesting because the pumped mode corresponds to $l=0$, while the side modes symmetrically expand as $l= \pm 1, \pm 2, \pm 3, \ldots$, where " + " and "-" stand, respectively, for higher and lower frequency side modes. The integer $l$ therefore becomes the only number that enables us to identify unambiguously the cavity modes of interest.

Unless otherwise specified, the parameters used in the whole article are the following: the polar eigennumber of the pumped mode is $\ell_{0}=14350$, corresponding to $\lambda_{0}=1560.5$ $\mathrm{nm}$ in vacuum; the refraction index is $n_{0}=1.43$ at $\lambda_{0}$, and the calcium fluoride cavity has a radius $a=2.5 \mathrm{~mm}$; the modal volume of the pumped cavity has been calculated to be equal to $V_{0}=6.6 \times 10^{-12} \mathrm{~m}^{3}$; the value of the Kerr coefficient is $n_{2}=3.2 \times 10^{-20} \mathrm{~m}^{2} / \mathrm{W}$; the cavity is critically coupled, with a loaded quality factor $Q_{0}=3 \times 10^{9}$, corresponding to a central modal bandwidth $\Delta \omega_{0} \simeq 2 \pi \times 64 \mathrm{kHz}$; and last, the polarization is TE.

\section{A. System below threshold}

Below threshold, the side modes are not excited so that they simply obey $\mathcal{A}_{ \pm l}=0$, with $l>0$. On the other hand, the fundamental mode $l=0$ is excited by the external pump, and from Eq. (27), it can be deduced that the electric field in this mode obeys

$$
\dot{\mathcal{A}}_{0}=-\frac{1}{2} \Delta \omega_{0} \mathcal{A}_{0}-i g_{0}\left|\mathcal{A}_{0}\right|^{2} \mathcal{A}_{0}+\frac{1}{2} \Delta \omega_{0} \mathcal{F}_{0} e^{i \sigma t},
$$

where

$$
\sigma=\Omega_{0}-\omega_{0}
$$

is the detuning angular frequency between the pump frequency and the cavity-mode resonance of the central mode. We can remove the explicit time dependence in this equation by introducing the variable transformation $\mathcal{B}_{0}=\mathcal{A}_{0} \exp [-i \sigma t]$ obeying

$$
\dot{\mathcal{B}}_{0}=-\frac{1}{2} \Delta \omega_{0} \mathcal{B}_{0}-i \sigma \mathcal{B}_{0}-i g_{0}\left|\mathcal{B}_{0}\right|^{2} \mathcal{B}_{0}+\frac{1}{2} \Delta \omega_{0} \mathcal{F}_{0} .
$$

Let us now consider that the steady state amplitude of the central mode is $\mathcal{A}_{0 s}$. Using the relationships $\dot{\mathcal{B}}_{0}=0$ and $\left|\mathcal{B}_{0}\right|=\left|\mathcal{A}_{0}\right|$, we can deduce that the input and modal number of photons are related by

$$
\left|\mathcal{F}_{0}\right|^{2}=\left[1+\frac{4 \sigma^{2}}{\Delta \omega_{0}^{2}}\right]\left|\mathcal{A}_{0 s}\right|^{2}+\frac{8 g_{0} \sigma}{\Delta \omega_{0}^{2}}\left|\mathcal{A}_{0 s}\right|^{4}+\frac{4 g_{0}^{2}}{\Delta \omega_{0}^{2}}\left|\mathcal{A}_{0 s}\right|^{6}
$$

in the steady state. This algebraic equation is bicubic so that for a given input $\left|\mathcal{F}_{0}\right|^{2}$, there may be one, two, or three solutions for $\left|\mathcal{A}_{0 s}\right|^{2}$. This kind of cubic equation leads the well-known hysteresis phenomenon, and in our case, it is worth recalling that when there are three solutions, the intermediate one is always unstable, therefore corresponding to a forbidden value.

It is essential here to identify these forbidden values because experimentally, the controllable (free) parameter is the external pump $\mathcal{F}_{0}$ and not the comb-generating internal field $\mathcal{A}_{0 s}$. Hence, from the nontrivial relationship between both, we need to establish a stability chart evidencing the forbidden values of the internal fields depending on the pumping. Such values appear in fact when the cubic dependence in Eq. (38) has local extrema, that is, when there are values of $\mathcal{A}_{0 s}$ for which the function

$$
\mathrm{C}=\frac{\partial\left[\left|\mathcal{F}_{0}\right|^{2}\right]}{\partial\left[\left|\mathcal{A}_{0 s}\right|^{2}\right]}
$$

is null; the forbidden values simply lie in between. This partial derivative function is a biquadratic function, and the hysteresis boundary exists wherever the related discriminant

$$
\Delta_{\text {hyst }}=64 \frac{g_{0}^{2}}{\Delta \omega_{0}^{4}}\left[\sigma^{2}-\frac{3}{4} \Delta \omega_{0}^{2}\right]
$$


is positive. In other words, stable values for the pump are those for which the condition $\mathrm{C}>0$ is fulfilled, explicitly leading to

$$
\left|\mathcal{A}_{0 s}\right|^{2} \notin\left[\mathrm{B}_{-}, \mathrm{B}_{+}\right]
$$

with the boundaries

$$
\mathrm{B}_{ \pm}=\frac{1}{g_{0}}\left[-\frac{2 \sigma}{3} \pm \frac{1}{3} \sqrt{\sigma^{2}-\frac{3}{4} \Delta \omega_{0}^{2}}\right] .
$$

The detuning condition for the existence of these boundaries is

$$
\sigma<-\frac{\sqrt{3}}{2} \Delta \omega_{0}=\sigma_{\text {hyst }} .
$$

When $\sigma>\sigma_{\text {hyst }}$, there is no hysteresis, and the central mode is stable regardless of its amplitude ( $\mathrm{C}$ is always positive in this case). This threshold detuning $\sigma_{\text {hyst }}$ below which forbidden values for $\mathcal{A}_{0 s}$ may arise is in fact significantly large, as it is even outside the limits $\pm \Delta \omega_{0} / 2$ delimiting the bandwidth of the pumped mode. It therefore appears that as long as the detuning is reasonable (e.g., within the bandwidth of the central mode), any value of the external field $\mathcal{F}_{0}$ only corresponds to one value of the internal field $\mathcal{A}_{0 s}$. Moreover, any value $\mathcal{A}_{0 s}$ can theoretically be reached and actually observed. For larger detunings below the resonance frequency $\omega_{0}$, hysteresis arises, and there are forbidden gaps for the internal modal amplitude.

Figure 4 shows how the stationary power $\mathcal{A}_{0 s}$ in the pumped mode varies when the laser power increases. In Fig. 4(a), the laser frequency is within the bandwidth of the central mode, and the modal power increases monotonously with the pump. We still have the same behavior even when the laser frequency

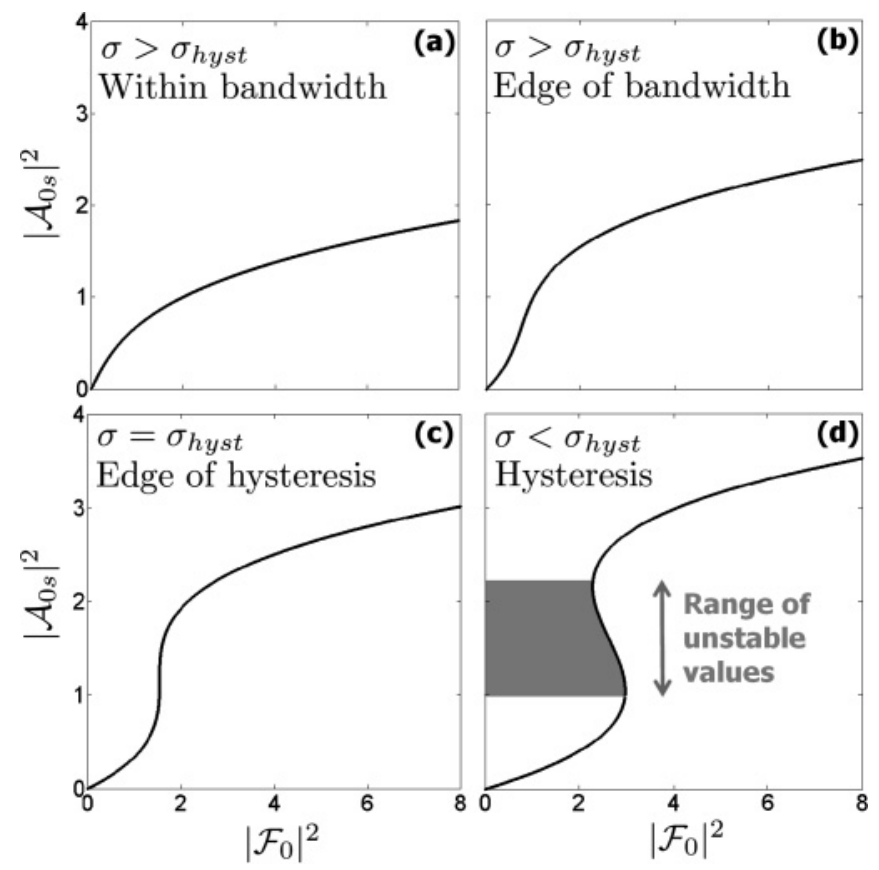

FIG. 4. Power $\left|\mathcal{A}_{0 s}\right|^{2}$ in the pumped mode as a function of the injected power $\left|\mathcal{F}_{0}\right|^{2}$, according to Eq. (38). Both variables are normalized in units of $\left|\mathcal{A}_{0}\right|_{\mathrm{th}}^{2}$. The various figures correspond to different laser detuning frequencies $\sigma$. These amplitude variations are valid as long as no comb is generated in the system. (a) $\sigma=0$; (b) $\sigma=-0.5 \Delta \omega_{0}$; (c) $\sigma=-0.86 \Delta \omega_{0}$; (d) $\sigma=-1.2 \Delta \omega_{0}$. is detuned to the edge of the bandwidth [in Fig. 4(b)]. However, when the laser frequency is detuned to $\sigma_{\text {hyst }} \simeq-0.86 \Delta \omega_{0}$, hysteresis arises. If the detuning is further increased leftward, there is a multivalued range where a single external pump power can lead to three possible different amplitudes for the central mode, as can be seen in Fig. 4(d). The intermediate solution is always unstable and can never be observed, while the other two are indeed stable: They can potentially generate a comb.

The central-mode amplitude $\mathcal{A}_{0 s}$ can also be viewed as the zeroth-order comb. In fact, this single-peaked comb is effectively observed when no side mode is excited, as, for example, below the comb-generation threshold $\left(\left|\mathcal{A}_{0}\right|^{2}=\right.$ $\left.\left|\mathcal{A}_{0 s}\right|^{2}\right)$; however, it becomes virtual above threshold when the central mode is depleted through FWM $\left(\left|\mathcal{A}_{0}\right|^{2}<\left|\mathcal{A}_{0 s}\right|^{2}\right)$. As we will further see, the concept of a zeroth-order comb is useful above threshold at the time to evaluate the efficiency of comb generation because the excess power $\left|\mathcal{A}_{0 s}\right|^{2}-\left|\mathcal{A}_{0}\right|^{2}$ corresponds to the number of photons originating from the pump. They are distributed among the various side modes through four-wave mixing. Hence computing the zerothorder comb provides a fast and accurate estimation of the number of photons available for the side modes and thus an estimation of their respective powers. The phenomenology below threshold being understood, we can now study the threshold conditions leading to comb generation whenever the central mode oscillation is stable.

\section{B. System at threshold}

For finding the threshold leading to oscillation for a given pair of side modes $\mathcal{A}_{ \pm l}=0$, a well-known technique is to investigate the linear stability of the trivial equilibrium $\mathcal{A}_{ \pm l}=0$. This equilibrium is perturbed with $\delta \mathcal{A}_{ \pm l}$, and the threshold is defined by the set of parameters separating the values for which the perturbation decays to 0 (the trivial equilibrium is stable) of those where the perturbation diverges to infinity (onset of side mode oscillations). In this stability analysis, no other modes than the specific pair $\delta \mathcal{A}_{ \pm l}$ are oscillating.

From Eq. (27), it can be shown that $\mathcal{A}_{0}$ still obeys Eq. (35), and then it still represents the so-called zeroth-order comb. On the other hand, the side mode perturbations obey

$$
\begin{aligned}
\delta \dot{\mathcal{A}}_{ \pm l}= & -\frac{1}{2} \Delta \omega_{ \pm l} \delta \mathcal{A}_{ \pm l}-i g_{0} \Lambda_{ \pm l}^{0, \mp l, 0} \mathcal{A}_{0}^{2} \delta \mathcal{A}_{\mp l}^{*} e^{i \varpi_{ \pm l} t} \\
& -i g_{0}\left[\Lambda_{ \pm l}^{ \pm l, 0,0}+\Lambda_{ \pm l}^{0,0, \pm l}\right]\left|\mathcal{A}_{0}\right|^{2} \delta \mathcal{A}_{ \pm l},
\end{aligned}
$$

where

$$
\varpi_{l}=2 \omega_{0}-\omega_{l}-\omega_{-l}=\varpi_{-l}
$$

is the modal detuning. This important parameter can also be viewed in this context as an overall (or cavity) dispersion parameter, simultaneously accounting for both geometrical and material dispersion. Note that the intermodal coupling coefficients $\Lambda_{l}^{0,-l, 0}, \Lambda_{l}^{l 00}$, and $\Lambda_{l}^{00 l}$ converge to 1 as $l \rightarrow 0$.

Once again, explicit time dependence should be removed: If we introduce the variables $\mathcal{B}_{0}=\mathcal{A}_{0} \exp [-i \sigma t]$ and $\delta \mathcal{B}_{ \pm l}=$ $\delta \mathcal{A}_{ \pm l} \exp \left[-i\left(\sigma+\frac{1}{2} \varpi_{ \pm l}\right) t\right]$, Eq. (44) can be explicitly rewritten 
as

$$
\left[\begin{array}{c}
\delta \dot{\mathcal{B}}_{l} \\
\delta \dot{\mathcal{B}}_{-l}^{*}
\end{array}\right]=\left[\begin{array}{cc}
M_{l} & R_{l} \\
R_{-l}^{*} & M_{-l}^{*}
\end{array}\right]\left[\begin{array}{c}
\delta \mathcal{B}_{l} \\
\delta \mathcal{B}_{-l}^{*}
\end{array}\right],
$$

with

$$
\begin{gathered}
M_{l}=-\frac{1}{2} \Delta \omega_{l}-i \sigma-\frac{1}{2} i \varpi_{l}-i g_{0}\left[\Lambda_{l}^{l 00}+\Lambda_{l}^{00 l}\right]\left|\mathcal{B}_{0}\right|^{2} \\
R_{l}=-i g_{0} \Lambda_{l}^{0,-l, 0} \mathcal{B}_{0}^{2} .
\end{gathered}
$$

When the central mode reaches the steady state $\mathcal{B}_{0 s}$, side modes are created when at least one of the eigenvalues $\lambda$ obeying the secular equation

$$
\left|\begin{array}{lc}
M_{l}-\lambda & R_{l} \\
R_{-l}^{*} & M_{-l}^{*}-\lambda
\end{array}\right|=0
$$

has a positive real part, which occurs concretely when

$$
\operatorname{Re}\left\{\left[M_{l}+M_{-l}^{*}\right]+\sqrt{\left[M_{l}-M_{-l}^{*}\right]^{2}+4 R_{l} R_{-l}^{*}}\right\}>0 .
$$

The preceding equation is the rigorous stability condition required for a side mode to appear at the exclusion of any other one. For practical purposes, we will consider some simplifying assumptions in order to move forward with this analysis. In particular, if we suppose that the first modes to reach threshold are relatively near the pump, some simplifications can be introduced. We can in this case consider that the intermodal coupling coefficients $\Lambda_{\eta}^{\alpha \beta \mu}$ are equal to 1 as close-by modes are almost perfectly overlapped; the confinement factors are also considered as identical and can be equated to 1 in first approximation. Along the same line, according to Eq. (28), the modal linewidth can also be considered as degenerated with $\Delta \omega_{0}$.

Provided that the argument of the square root in Eq. (49) is positive (if it is negative, the inequality is automatically false), the stability condition for a side mode pair $\pm l$ can be rewritten as $\mathrm{S}(l)<0$, with

$$
\begin{aligned}
\mathrm{S}(l)= & 12\left[g_{0}\left|\mathcal{A}_{0 s}\right|^{2}\right]^{2}+8\left[2 \sigma+\varpi_{l}\right]\left[g_{0}\left|\mathcal{A}_{0 s}\right|^{2}\right] \\
& +\left[2 \sigma+\varpi_{l}\right]^{2}+\Delta \omega_{0}^{2} .
\end{aligned}
$$

This stability condition may provide qualitatively different behaviors depending on whether dispersion is taken into account through $\varpi_{l}$. In order to gain a simpler understanding of the threshold phenomenology, we will first neglect dispersion to calculate the threshold power for comb generation.

\section{Neglecting cavity dispersion}

Within this approximation, we set $\varpi_{l}=0$, and the stability condition of Eq. (50) no longer depends on $l$ so that all the modes near the pump (where the modal gains, volumes, confinement factors, etc., are nearly degenerate) become stable or turn unstable simultaneously. Note that the analysis performed in this section is also valid when geometrical and material dispersion cancel each other without being negligible themselves since in that case, we still have $\varpi_{l}=0$.

Equation (50) is quadratic in $g_{0}\left|\mathcal{A}_{0 s}\right|^{2}$ : It is therefore a bottom-down parabola that may or may not intersect the abscissa axis, depending on the various parameters. If it does not, the pair of side modes $\pm l$ stays in the trivial equilibrium and is not excited by the pump; if it does, these side modes $\pm l$ oscillate for the parameter range for which the parabola is below the abscissa axis.

The zeros of Eq. (50) constitute a boundary curve that can be formally expressed as

$$
\tilde{\mathrm{B}}_{ \pm}=\frac{1}{g_{0}}\left[-\frac{2 \sigma}{3} \pm \frac{1}{3} \sqrt{\sigma^{2}-\frac{3}{4} \Delta \omega_{0}^{2}}\right],
$$

corresponding, respectively, to the upper $(+)$ and lower (-) power boundary values leading to comb generation. The stability condition $S>0$ for comb generation therefore translates explicitly into

$$
\left|\mathcal{A}_{0 s}\right|^{2} \in\left[\tilde{\mathrm{B}}_{-}, \tilde{\mathrm{B}}_{+}\right] .
$$

For these power boundaries to be real and positive, the discriminant associated with the quadratic equation should be positive, and the detuning $\sigma$ should be negative. In particular, since the absolute threshold corresponds to the parabola touching the abscissa axis, the quadratic equation should have a null discriminant, thereby leading to the following threshold value for the detuning:

$$
\sigma_{c r}=-\frac{\sqrt{3}}{2} \Delta \omega_{0} .
$$

The value

$$
\left|\mathcal{A}_{0}\right|_{c r}^{2}=\frac{1}{\sqrt{3}} \frac{\Delta \omega_{0}}{g_{0}}
$$

therefore corresponds to the critical number of photons in the pumped mode leading to comb generation when the detuning frequency $\sigma$ is varied.

The range of values leading to solutions lying between the zeros defined in Eq. (51) leads to sustained comb generation. Geometrically, it corresponds to the parabola intersecting the abscissa axis: Stable comb generation occurs for parameters lying within the closed surface delimited by the parabola and the axis. Algebraically, comb generation corresponds to the situation in which the aforementioned discriminant is strictly positive, therefore leading to the phase-detuning condition

$$
\sigma<\sigma_{c r} .
$$

The detuning therefore has to be very large (outside the bandwidth) to trigger comb generation.

Further analysis shows that the absolute minimum power leading to comb generation can be obtained through the equation $\partial \tilde{\mathrm{B}}_{-} / \partial \sigma=0$, which yields the detuning and the power for which the lower boundary branch reaches a minimum. The optimum detuning is found to be

$$
\sigma_{\mathrm{opt}}=-\Delta \omega_{0}
$$

and leads to the following threshold power for comb generation:

$$
\left|\mathcal{A}_{0}\right|_{\mathrm{th}}^{2}=\frac{1}{2} \frac{\Delta \omega_{0}}{g_{0}}=\frac{1}{2 \hbar \omega_{0}} \frac{n_{0}^{2}}{n_{2} c} \frac{V_{0}}{Q_{0}} .
$$

It can therefore be deduced that $\left|\mathcal{A}_{0}\right|_{c r}^{2}=[2 / \sqrt{3}]\left|\mathcal{A}_{0}\right|_{\text {th }}^{2}$, meaning that the critical power as the laser frequency $\sigma$ is detuned is nearly $15 \%$ higher than the absolute threshold value. We will consider the threshold power $\left|\mathcal{A}_{0}\right|_{\text {th }}^{2}$ as a normalization parameter throughout this article. 


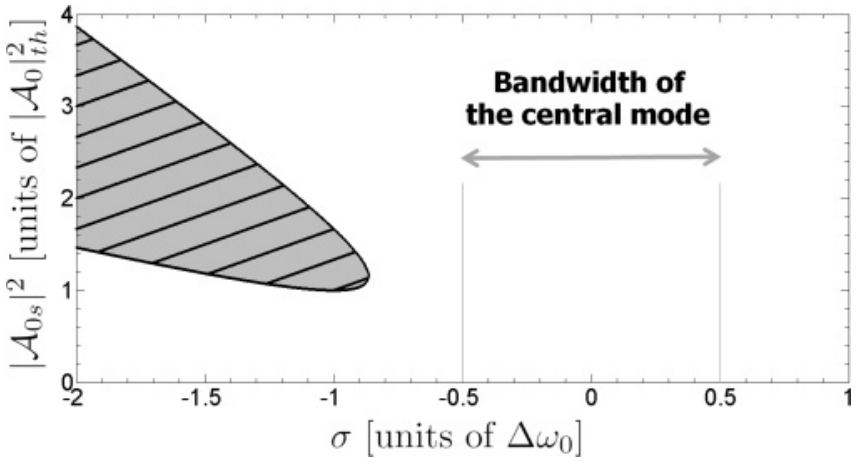

FIG. 5. Stability chart for the pumped mode and for the combs as a function of the laser detuning $\sigma$ and the stationary pumped mode power $\left|\mathcal{A}_{0 s}\right|^{2}$ in the zero-dispersion case. The shaded area corresponds to unstable values of $\left|\mathcal{A}_{0 s}\right|^{2}$ [values destabilized by hysteresis, as explained in Fig. 4(c)] defined by Eq. (41). On the other hand, the hatched area corresponds to values of the pumped mode leading to stable comb generation, according to Eq. (52). Hence comb generation is only possible wherever the hatched area does not overlap the shaded area. Since both areas are perfectly overlapped, comb generation is impossible in the zero-dispersion case.

Surprisingly, we find that $\sigma_{c r}=\sigma_{\text {hyst }}$; that is, detuning needed for comb generation is also the minimal detuning for which $\mathcal{A}_{0 s}$ is unstable. Moreover, the boundaries defined by Eqs. (51) and (42) are identical because we have $\tilde{B}_{ \pm} \equiv \mathrm{B}_{ \pm}$; therefore the stability area for the side modes perfectly overlaps the instability area for $\mathcal{A}_{0 s}$, as it can be seen in Fig. 5. In other words, the simultaneous fulfillment of both Eqs. (41) and (52) is impossible as the side modes are supposed to become stable exactly when the fundamental mode that is exciting them loses its stability. Therefore this analysis shows that in a medium without dispersion (or equivalently, in a medium where geometrical and material dispersions perfectly cancel each other), frequency comb-generation cannot arise. This result is highly nonintuitive as the zero-dispersion limit is sometimes presented as the ideal situation that would allow for virtually unlimited wide-span comb generation. However, it is important to note that the degeneracy between these two areas can eventually be lifted by other phenomena that have not been considered in our analysis, such as thermal effects, or eventually, some spatiotemporal effects that have not been taken into account in our modal expansion model.

We will show in the next section that material and geometrical dispersion are not detrimental to WGM comb generation. In fact, they are necessary to permit the existence of stable combs. They can also limit their frequency span. The existence of dispersion will explain why the first pair of side modes to reach the oscillation threshold is not necessarily the one adjacent to the pump and also why it is possible to generate combs with multiple-FSR spacing, as it has been reported in the literature [6,7].

\section{EFFECT OF MATERIAL AND GEOMETRICAL DISPERSION}

In this section, we analyze the effect of dispersion on the threshold and stability of optical combs. Here the dispersion term $\varpi_{l}$ becomes relevant in Eq. (50).
Equation (50) is still a bottom-down parabola, but now its coefficients depend on the mode order $l$. Following the same reasoning as in the preceding subsection, it is found that the upper and lower power bounds leading to $\pm l$ side mode oscillation are

$$
\mathrm{B}_{ \pm}(l)=\frac{1}{g_{0}}\left[-\frac{2 \sigma_{l}}{3} \pm \frac{1}{3} \sqrt{\sigma_{l}^{2}-\frac{3}{4} \Delta \omega_{0}^{2}}\right],
$$

with

$$
\sigma_{l}=\sigma+\frac{1}{2} \varpi_{l}<\sigma_{c r}=-\frac{\sqrt{3}}{2} \Delta \omega_{0} .
$$

It is found that the threshold power for comb generation can be obtained through $\partial \mathrm{B}_{-}(l) / \partial \sigma_{l}=0$ and yields the same value as in Eq. (57), that is, it is the same as when there is no dispersion. It is also the same for all the modes near the pump. However, there is an essential difference as far as critical and optimal phase detunings for each pair $\pm l$ are concerned: The formulas are the same as in Eqs. (55) and (56), except that $\sigma$ has to be replaced by $\sigma_{l}$.

Understanding how dispersion affects comb generation requires an explicit formulation of the dispersion parameter $\varpi_{l}$ as a function of the side mode orders $l$. We will discuss this in the next section.

\section{A. Explicit determination of material and geometrical dispersion}

In order to evaluate the dispersion parameter $\varpi_{l}$, it is useful to Taylor-expand Eq. (14) at up to order $l^{2}$ following

$$
\omega_{l}=\omega_{0}+\frac{c}{n\left(\omega_{l}\right) a} l+\delta \omega_{l}
$$

with

$$
\delta \omega_{l} \simeq 2^{-\frac{1}{3}} \ell_{0}^{-\frac{2}{3}} \xi_{1} \frac{c}{n_{0} a}\left[l-\frac{1}{9 \ell_{0}} l^{2}\right] .
$$

The parameter $\varpi_{l}$ can be decomposed into geometrical and material dispersion following

$$
\begin{aligned}
\varpi_{l} & =\left.\varpi_{l}\right|_{n=n_{0}}+\left.\varpi_{l}\right|_{\delta \omega_{l}=0} \\
& \equiv \varpi_{\mathrm{geo}, l}+\varpi_{\mathrm{mat}, l} .
\end{aligned}
$$

From the definition of Eqs. (45) and (60), the geometrical dispersion can be straightforwardly obtained as

$$
\varpi_{\mathrm{geo}, l} \simeq\left[\xi_{1} \frac{2^{\frac{2}{3}}}{9} \frac{c}{n_{0} a} \ell_{0}^{-\frac{5}{3}}\right] l^{2} .
$$

It appears that geometrical dispersion increases quadratically with the reduced mode order. Since $l$ is a measure of the spectral distance, this term indicates that geometrical dispersion for a symmetric pair of side modes is exclusively of second order. A deviation from this law is to be expected for waveguides that are not exactly spherical. However, this geometrical dispersion is still generally normal.

As far as material dispersion is concerned, the first step is to Taylor-expand the modal wave number at up to the second order following

$$
\frac{\omega_{l}}{c} n\left(\omega_{l}\right)=\frac{\omega_{0}}{c} n_{0}+\beta_{\omega_{0}}^{\prime}\left[\omega_{l}-\omega_{0}\right]+\frac{1}{2} \beta_{\omega_{0}}^{\prime \prime}\left[\omega_{l}-\omega_{0}\right]^{2},
$$


where

$$
\beta_{\omega_{0}}^{\prime}=\frac{1}{c}\left[n_{0}+\omega_{0} n_{\omega_{0}}^{\prime}\right] \quad \beta_{\omega_{0}}^{\prime \prime}=\frac{1}{c}\left[2 n_{\omega_{0}}^{\prime}+\omega_{0} n_{\omega_{0}}^{\prime \prime}\right]
$$

are, respectively, the inverse group velocity and the group velocity dispersion in the medium. These parameters can be computed for any frequency using the Sellmeier expansion $[27,28]$. From Eq. (60), we have $\left[\omega_{l} n\left(\omega_{l}\right)-\omega_{0} n_{0}\right] / c \simeq l / a$, and therefore the physical solution of Eq. (64) can be shown to depend on material dispersion according to

$$
\omega_{l}=\omega_{0}+\frac{1}{\beta_{\omega_{0}}^{\prime \prime}}\left[-\beta_{\omega_{0}}^{\prime}+\sqrt{\beta_{\omega_{0}}^{\prime 2}+2 \beta_{\omega_{0}}^{\prime \prime} \frac{l}{a}}\right] .
$$

Hence combining Eqs. (60) and (66) yields the following expression for material dispersion:

$$
\varpi_{\mathrm{mat}, l} \simeq \frac{\beta_{\omega_{0}}^{\prime \prime}}{a^{2} \beta^{\prime 3}} l^{2} .
$$

Finally, we find that the overall dispersion varies quadratically with the mode order following

$$
\varpi_{l}=-\zeta l^{2}
$$

with

$$
\begin{aligned}
\zeta & =-\left[\varpi_{\mathrm{geo}, 1}+\varpi_{\mathrm{mat}, 1}\right] \\
& \simeq-0.41\left[\frac{c}{n_{0} a}\right]^{\frac{8}{3}} \omega_{0}^{-\frac{5}{3}}-\frac{2 n_{\omega_{0}}^{\prime}+\omega_{0} n_{\omega_{0}}^{\prime \prime}}{n_{0}}\left[\frac{c}{n_{0} a}\right]^{2} .
\end{aligned}
$$

This real coefficient $\zeta$ determines the cavity (overall) dispersion behavior for the side modes, and it can be either positive or negative, corresponding, respectively, to anomalous or normal dispersion. It depends on the pumping frequency $\omega_{0}$; on the optical properties of the bulk resonator through through $n_{0}, n_{\omega_{0}}^{\prime}$, and $n_{\omega_{0}}^{\prime \prime}$; and on its characteristic size $a$. It can therefore be viewed as a constant parameter of the pumped resonator, fixed for a given resonator and pumping frequency.

We emphasize that one can use the Sellmeier expansion to determine the refractive index and its derivatives and then the parameter $\zeta$ using Eq. (69). However, earlier works have indicated that there is sometimes a discrepancy between theoretical and experimental data (see, e.g., Ref. [29]). The fact that $n_{\omega_{0}}^{\prime \prime}$ changes its sign near $1550 \mathrm{~nm}$ for calcium fluoride, moreover, gives critical importance to third- and fourth-order dispersion. This may dramatically increase the aforementioned discrepancy. It is speculated that the discrepancy between dispersion values derived from Sellmeier expansion and those obtained for experiments in WGM resonators may originate from variations of the dispersion properties of the material according to fabrication processes or dependence of dispersion with light power, either directly (if irradiance changes the refraction index) or indirectly (through thermal lensing). The Sellmeier expansion can therefore only approximate the real value of $\zeta$.

The main conclusion of the preceding analysis is that an optical-frequency comb is generated when both the conditions $\mathrm{C}>0$ for the central mode and $\mathrm{S}(l)<0$ for at least one side mode pair are fulfilled simultaneously. Explicitly, this double condition can be rewritten as

$$
\begin{gathered}
\text { Stability for } l=0:\left|\mathcal{A}_{0 s}\right|^{2} \notin\left[\mathrm{B}_{-}(0), \mathrm{B}_{+}(0)\right], \\
\text { Stability for } l \neq 0:\left|\mathcal{A}_{0 s}\right|^{2} \in\left[\mathrm{B}_{-}(l), \mathrm{B}_{+}(l)\right],
\end{gathered}
$$

where the stability boundaries $\mathrm{B}_{ \pm}(l)$ are defined in Eq. (58). Different phenomenologies are to be expected according to the sign and amplitude of $\zeta$, as hereinafter discussed.

\section{B. Case $\zeta<0$ (normal cavity dispersion)}

The critical detuning condition of Eq. (59) leads to the stability conditions

$$
\begin{aligned}
\sigma & <\sigma_{c r}, \\
|l| \leqslant l_{\max }(\sigma) & =\sqrt{(2 / \zeta)\left[\sigma-\sigma_{c r}\right]} .
\end{aligned}
$$

Hence, in this case, very strong detunings are needed for comb generation (because $\sigma_{c r}$ is negative), in any case outside the bandwidth of the fundamental mode. Moreover, there is a maximal side mode order beyond which direct comb generation from the pump is impossible. This case is represented in Fig. 6(a), where it can be seen that according to Eq. (58), the hatched area standing for stable comb generation moves leftward, thereby lifting the degeneracy with the area of unstable central mode. Note that in practice, the numerical value of $l_{\max }$ has to be rounded to the lower integer value.

However, comb generation in this case is still very unlikely for two reasons. The first one is that, as it can be seen in Fig. 6(a), generating a comb with normal dispersion would

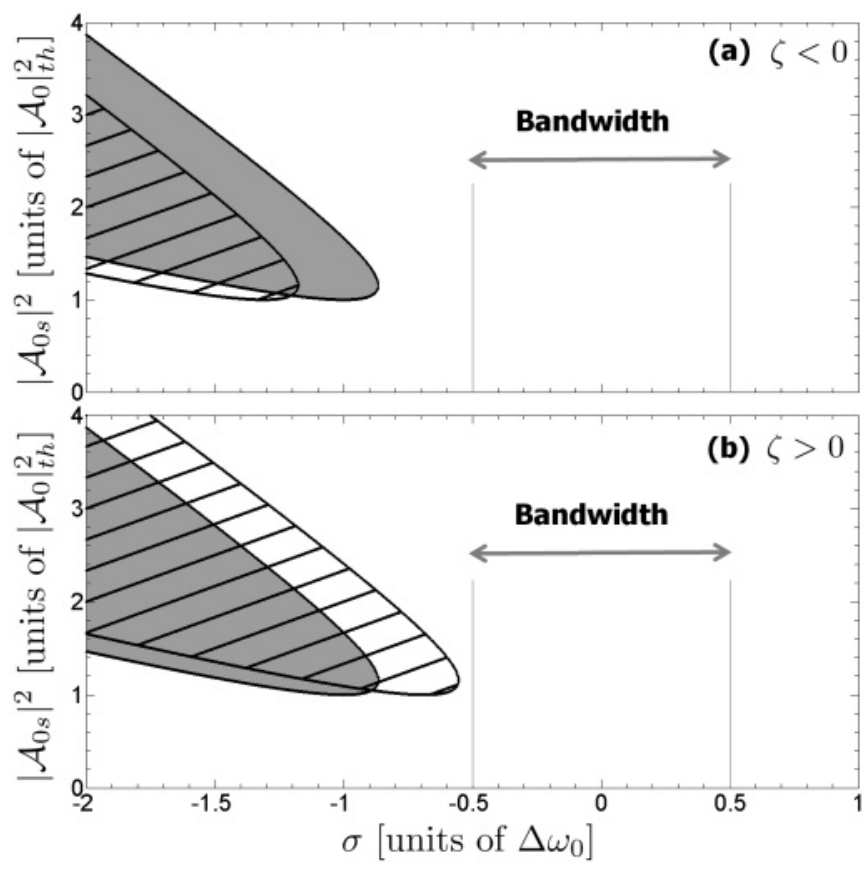

FIG. 6. Stability diagram for comb generation for the side modes $l= \pm 10$. The shaded region still represents forbidden (unstable) values of the pumped mode, while the hatched areas correspond to those where a stable pump can effectively generate the comb. Stable comb generation effectively occurs in the hatched and nonshaded areas. Note that the shaded area does not change, but the hatched does. (a) Normal cavity dispersion $\zeta=-2 \pi \times 400 \mathrm{~Hz}$. (b) Anomalous cavity dispersion $\zeta=2 \pi \times 400 \mathrm{~Hz}$. 
require us to detune the laser very far away from the modal bandwidth, and independently of the side mode pair $\pm l$, the detuning would in any case be stronger than $\sigma_{c r}$, which is yet significantly out of the bandwidth. Pumping the cavity with such a strongly detuned laser input is not experimentally realistic and can hardly be implemented. The second reason is that even if this could be done, Fig. 6(a) also shows that the stable comb generation area is below the instability range so that it is the lowest amplitude solution of the hysteresis area that should be excited. Unfortunately, this solution is too close to the instability area and is therefore only weakly stable. The system will preferably jump to the highest amplitude solution, which is far from from the instability zone and much more stable. Hence the low-amplitude solution needed for comb generation is not likely to be effectively sustained. Therefore the case of normal dispersion is not interesting for comb generation, and it will not be considered further in this article.

\section{Case $\zeta>0$ (anomalous cavity dispersion)}

Here the critical detuning condition of Eq. (59) gives

$$
\begin{gathered}
\sigma>\sigma_{c r}, \\
|l| \geqslant l_{\min }(\sigma)=\sqrt{(2 / \zeta)\left[\sigma-\sigma_{c r}\right]}
\end{gathered}
$$

The numerical value of $l_{\min }$ has to be rounded to the higher integer value. The preceding equation indicates that there is a minimal side mode order below which direct comb generation from the pump is impossible. On the other hand, the hatched area standing for stable comb generation moves rightward in Fig. 6(b), according to Eq. (58). Hence, in the case of anomalous dispersion, this area can also be quite far away from the hysteresis zone, meaning that central mode power may be unconditionally stable and thus experimentally observable. Therefore anomalous dispersion can lead to comb generation. This phenomenology can be related to the so-called dispersive bistability analyzed in Ref. [30].

Figure 7 shows that for some side mode pairs, this dispersion-induced shift may be significant enough to allow for stable comb generation when the laser detuning $\sigma$ is within the cavity-mode bandwidth (where there is no hysteresis; increasing the laser power in this area corresponds to increasing $\left|\mathcal{A}_{0 s}\right|^{2}$; see Fig. 4). For example, the pair $l= \pm 30$ appears to have a wider stability area than the pair $l= \pm 20$. For a fixed detuning within the bandwidth, the corresponding threshold power is higher. If the laser power is slowly swept upward, the pair \pm 20 will oscillate before the pair \pm 30 . Figure 7 also shows that increasing the laser input power too much leads to instability in both cases. For example, if the central mode is 10 times above threshold and the laser is perfectly resonant $(\sigma=0)$, none of these two pairs will oscillate. This is a quite counterintuitive conclusion as one would think that increasing the pump power should progressively excite more modes and enhance their stability but not quench them.

The preceding analysis illustrates that the stability behavior of the combs is nontrivial. Not all the side modes become stable or unstable simultaneously. We will show in the next section that this modal discrimination in terms of stability explains the phenomenon of comb versatility.

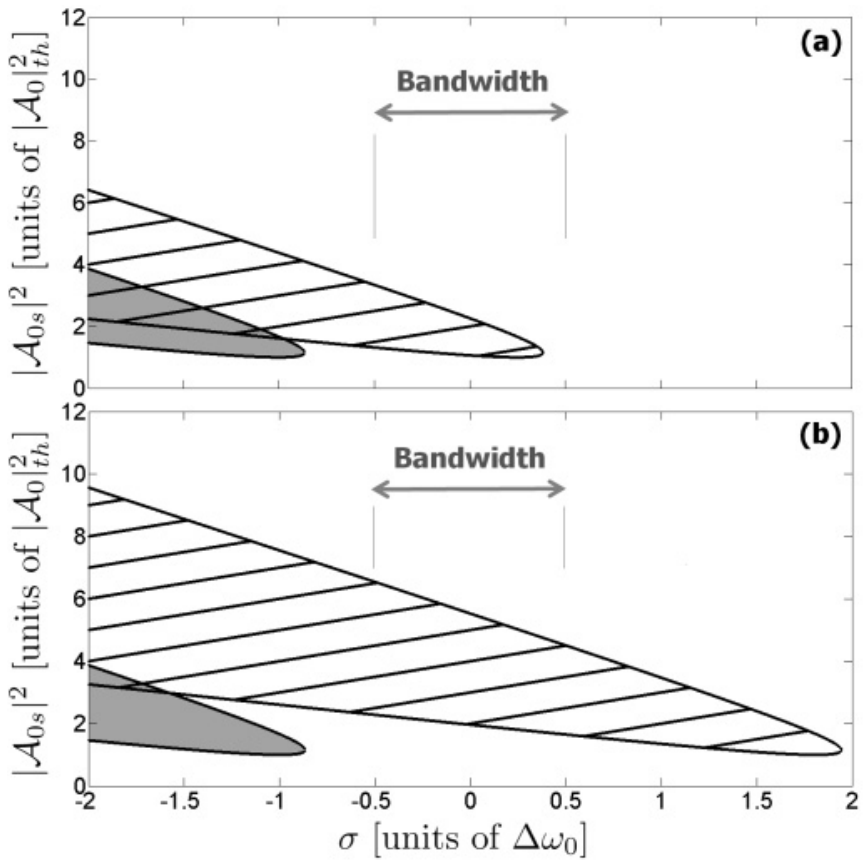

FIG. 7. Stability diagram of various pairs of side modes in the anomalous dispersion regime, with $\zeta=2 \pi \times 400 \mathrm{~Hz}$. Hatched and shaded areas have the same meaning as in Fig. 6, and this figure can be interpreted as a follow-up of Fig. 6(b) when the side mode order increases: (a) $l= \pm 20$; (b) $l= \pm 30$.

\section{Comb versatility for anomalous dispersion}

The stability diagrams of Figs. 6 and 7 were plotted in the plane $\sigma-\left|\mathcal{A}_{0 s}\right|^{2}$ for given side mode pairs $(l= \pm 10, \pm 20$, and $\pm 30)$. They were useful in order to show how dispersion lifts the degeneracy between the stable area for comb generation and the unstable area for the central-mode power. It is also insightful to plot the stability diagram in the plane $l-\left|\mathcal{A}_{0 s}\right|^{2}$ for a fixed value of the laser detuning $\sigma$. The interest here is that we can see the modes that could be directly excited by the pump (once the laser frequency has been set).

Figure 8 displays an example of such a stability diagram, where the laser input is resonant with the central mode $(\sigma=0)$. The solid curves are plotted according to Eq. (58) when $l$ is varied. It can be seen that for these parameters, no mode of order $|l|<l_{\min }=17$ can be excited (value rounded upward from the exact $l_{\min }=16.65$ ), as expressed in Eq. (72). However, the pair $|l|=l_{\min }$ is not the first to be excited when the power is increased. The oscillating modes $|l|=l_{\text {th }}$ at threshold are obtained as the solution of the equation $\partial \mathrm{B}_{-}(l) / \partial l=0$ and are found to correspond to $\sigma_{l}=\sigma_{\mathrm{opt}}$, yielding

$$
|l|=l_{\mathrm{th}}(\sigma)=\sqrt{(2 / \zeta)\left[\sigma-\sigma_{\mathrm{opt}}\right]}
$$

The preceding value has to be rounded to the nearest integer, and in our case, we obtain $l_{\text {th }}=18$ (rounded from 17.89).

The stability diagram of Fig. 8 also indicates that only symmetrical bands of modes can be excited, far away from the central mode. For example, if the central mode is pumped twice above threshold $\left[\left|\mathcal{A}_{0 s}\right|^{2}=2\left|\mathcal{A}_{0}\right|_{\text {th }}^{2}\right]$, then only the modes such that $19<|l|<30$ can be excited by the pump. This is precisely the mechanism that explains earlier results where 


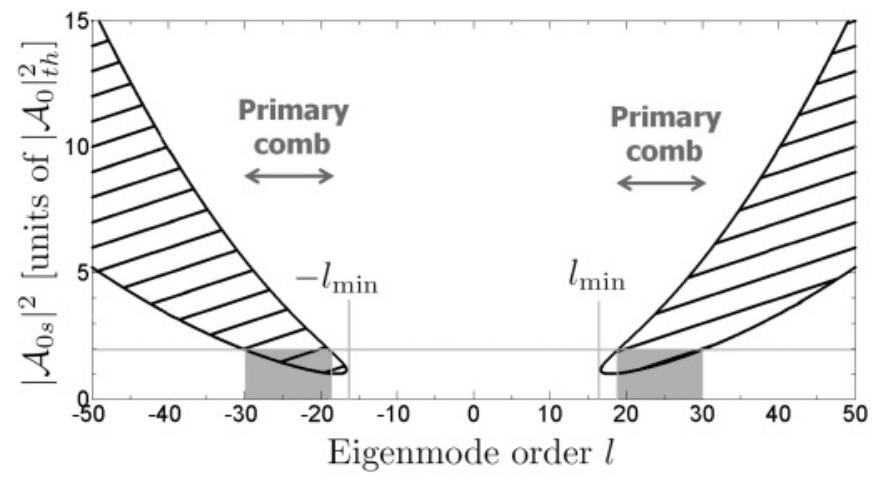

FIG. 8. An example of a stability diagram for comb generation as a function of eigenmode order $l$ and power $\left|\mathcal{A}_{0 s}\right|^{2}$ in the central mode. The dispersion parameter is set to $\zeta=2 \pi \times 400 \mathrm{~Hz}$ and the laser detuning to $\sigma=0$. The hatched area corresponds to stable comb generation, and the shaded areas are only there to emphasize the intervals hereinafter discussed. If the central mode is pumped twice above threshold, only the modes fulfilling $19<|l|<30$ can be excited. No mode of order $|l|<l_{\min }=17$ can be excited here through degenerate FWM.

combs with multiple FSR spacing were observed: It is the consequence of the fact that the modes adjacent to the pump are not directly excited by the pump, while some spectrally distant modes in a narrow frequency range experience parametric gain. Conversely, if we want to know what power in the central mode is required to excite the modes $l= \pm 40$, the figure shows that the answer is between 3.2 and 10 times the threshold (this indicates that a given pair of modes is not excited for any value of the pump).

Figure 9 shows how the stability areas are shifted when the laser frequency is detuned from one edge of the bandwidth to the other, passing through the resonance frequency. Equation (72) indicates that the minimum mode $l_{\min }$ under which no mode can be excited by the pump increases with the detuning frequency $\sigma$. Hence, for a fixed pump power, detuning the laser frequency positively would shift the stability bands for $|l|$ toward infinity.

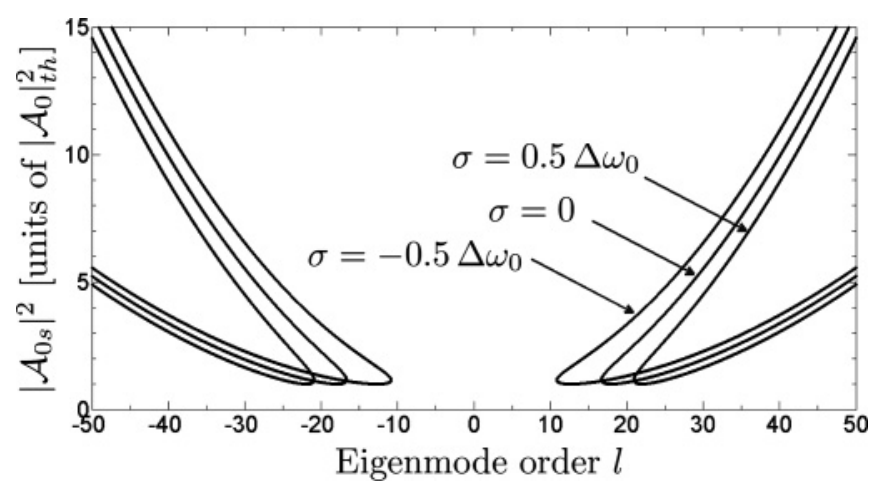

FIG. 9. Various stability diagrams for comb generation as a function of eigenmode order $l$ and power $\left|\mathcal{A}_{0 s}\right|^{2}$ in the central mode, as the laser detuning $\sigma$ is varied. The dispersion parameter is still set to $\zeta=2 \pi \times 400 \mathrm{~Hz}$, and the curve corresponding to $\sigma=0$ is exactly the same as the one in Fig. 8. The hatchings have been omitted in this figure in order to improve its clarity.
These stability diagrams are consistent with the experimental observations of comb versatility in WGMs reported so far. In particular, the FSR order of the comb was monitored in Ref. [6] through laser frequency detuning. This method corresponds to shifting the stability diagram, as shown in Fig. 9. On the other hand, tuning the comb FSR order through the pump power and/or the coupling conditions (i.e., $\Delta \omega_{0}$ ) is the method that was used in Ref. [7]. It corresponds to the stability diagram of Fig. 8.

\section{E. Notion of primary comb}

We have just shown that comb generation in WGMs is a cascading process. When the cavity is pumped above threshold, the first modes to oscillate are directly excited by the pump. We refer to these modes as the first-order comb, or the primary comb (see Fig. 8). The primary comb is therefore constituted with the oscillating side modes whose amplitudes are exclusively due to the pump, while their phases may be affected by other side modes (which are themselves exclusively pump induced). In other words, all the photons that are in the side modes $\pm l$ originate from the pump through the photonic interaction $2 \hbar \omega_{0} \rightarrow \hbar \omega_{l}+\hbar \omega_{-l}$, while the pump is depleted accordingly but also receives back some photons through $\hbar \omega_{l}+\hbar \omega_{-l} \rightarrow 2 \hbar \omega_{0}$. The pump and the modes of the primary comb will subsequently interact to excite new modes, thereby creating the secondary comb. The process follows on until a steady state is reached. The overall comb is therefore made of the primary and all the subsequent higher order combs.

It is important to note that from an experimental point of view, the power increase is performed through the external field power $\left|\mathcal{F}_{0}\right|^{2}$. However, from a physical perspective, the most relevant parameter for comb generation is the power inside the central mode, which corresponds to $\left|\mathcal{A}_{0 s}\right|^{2}$ [obeying Eq. (35)]. This is due to the fact that all the stability charts have been established relative to $\left|\mathcal{A}_{0 s}\right|^{2}$. Moreover, this control variable is particularly suitable because as emphasized earlier, the difference $\left|\mathcal{A}_{0 s}\right|^{2}-\left|\mathcal{A}_{0}\right|_{\text {th }}^{2}$ gives a good approximation of the number of photons that are available to the various side modes. When $\left|\mathcal{A}_{0 s}\right|^{2}$ is given, it is easy to determine the corresponding $\left|\mathcal{F}_{0}\right|^{2}$ through Eq. (38); for example, if $\left|\mathcal{A}_{0 s}\right|^{2}=x$ in units of threshold power, then $\left|\mathcal{F}_{0}\right|^{2}=x^{3}+x$ in the same units for $\sigma=$ 0 . Hence pumping the cavity just above threshold $\left(\left|\mathcal{A}_{0 s}\right|^{2}=\right.$ 1.01) requires $\left|\mathcal{F}_{0}\right|^{2}=2.04$, but reaching $\left|\mathcal{A}_{0 s}\right|^{2}=3$ requires 15 times more power from the external pump, as $\left|\mathcal{F}_{0}\right|^{2}=30$.

\section{COMB DYNAMICS}

The dynamics of the comb is numerically investigated by simulating the rate equations (27). The nonlinear terms induced by FWM effectively affect the modal dynamics only when they are resonant, that is, when the detuning frequencies $\varpi_{\alpha \beta \mu \eta}$ are smaller than the bandwidth $\Delta \omega_{\eta}$. The resonant terms are those fulfilling both the conservation of energy and angular momentum. Therefore the relevant terms of the FWM sum in the equation for $\mathcal{A}_{\eta}$ are exclusively those involving modes $\alpha$, $\beta$, and $\mu$ fulfilling $l_{\alpha}-l_{\beta}+l_{\mu}=l_{\eta}$.

We have shown in Fig. 3 that modes of 1000 free spectral ranges apart are still very well overlapped. Since we are only considering 201 modes, the modes are spatially 
quasidegenerated. We set $\Lambda_{\eta}^{\alpha \beta \mu}=1$ for the sake of simplicity as well as $\Delta \omega_{\eta} \equiv \Delta \omega_{0}$. On the other hand, we find from Eqs. (60), (61), and (66) that the intermodal detuning can be explicitly expressed as a function of dispersion and side mode order following

$$
\varpi_{\alpha \beta \mu \eta}=\frac{1}{2} \zeta\left[l_{\alpha}^{2}-l_{\beta}^{2}+l_{\mu}^{2}-l_{\eta}^{2}\right] .
$$

Hence the dispersion phenomena in the cavity are still governed by the single parameter $\zeta$. This quadratic expression of the intermodal detuning is still valid as long as the span of the comb is restricted to a spectral range where the cavity dispersion is well approximated with only a second-order expansion.

The rate equations (27) have been numerically simulated using a fourth-order Runge-Kutta algorithm. A total of 201 modes are considered simultaneously $(-100 \leqslant l \leqslant 100)$, and it corresponds to a wavelength span of $\sim 20 \mathrm{~nm}$. The initial conditions are the vacuum fluctuations: They are randomly distributed with $\left\langle\mathcal{A}_{l}(0)\right\rangle=0$ and $\left\langle\left|\mathcal{A}_{l}(0)\right|^{2}\right\rangle=1 / 2$, where $\langle\cdot\rangle$ stands for the ensemble average over $l$. For each comb, we simulated the multimode dynamics for a total duration of $T=$ $2 \mathrm{~ms}$, where the steady state is reached. The combs displayed here are histograms of the modal power power plotted as a function of the eigenmode order. The corresponding figures can be viewed as fast-scale Fourier spectra.

\section{A. Spectrum of the comb for various pump powers}

Figure 10(a) displays the full comb just above threshold, when the cavity is pumped only $1 \%$ above threshold. We can observe that the pump only generates a single side mode pair, whose order is $l_{\text {th }}= \pm 18$, as theoretically predicted in Eq. (73). When the pump power is increased in Fig. 10(b), the primary comb is still constituted with only one mode pair $(l= \pm 19)$, which is taking all the energy available from the pump through

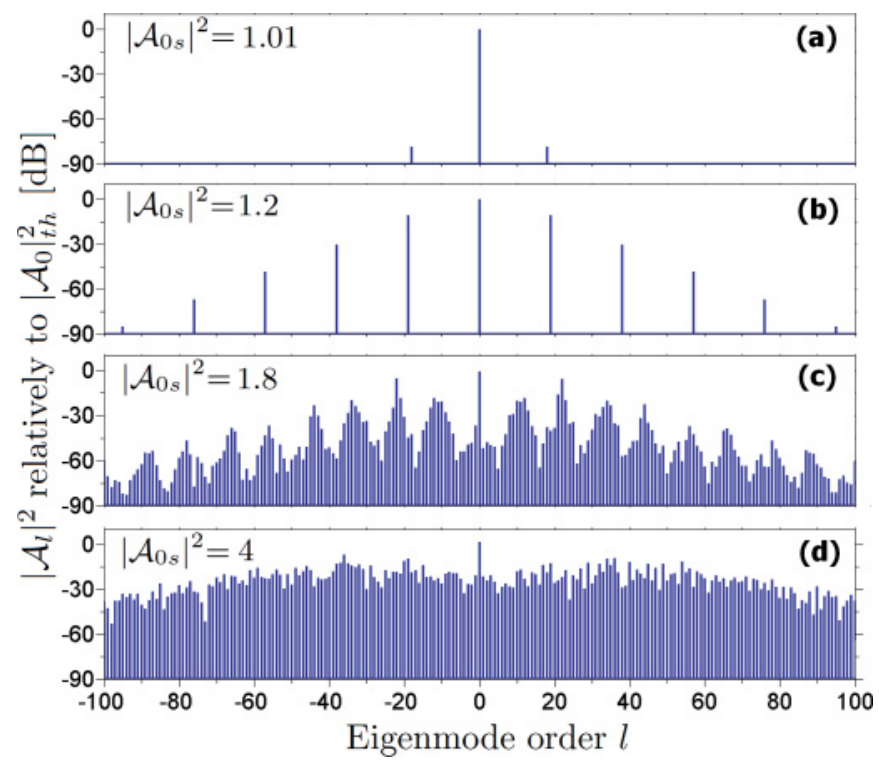

FIG. 10. (Color online) Evolution of the full comb as the pump power in the central mode is increased. The displayed combs correspond to spectrum snapshots at $t=2 \mathrm{~ms}$. The parameters are $\zeta=2 \pi \times 400 \mathrm{~Hz}$ and $\sigma=0$. The $\left|\mathcal{A}_{0 s}\right|^{2}$ is expressed in units of the threshold power $\left|\mathcal{A}_{0}\right|_{\text {th }}^{2}$. multimode competition $[31,32]$. The interaction between the pump and this oscillating side mode generates new modes that are spectrally located at integer multiples of the primary comb $(|l|=19,38,57$, etc. $)$. In this case, the secondary and higher order combs are harmonic relative to the primary comb. These spectra do in fact correspond to combs with multiple FSR spacing, as experimentally observed in Refs. [6,7]. It is also interesting to note that this harmonic excitation of side modes somehow corresponds to the bichromatic pumping scheme investigated in Ref. [33], and it was shown to be a thresholdless process. The pump power is further increased in Fig. 10(c); we can observe that a subharmonic higher order comb arises, owing to the emergence of stability bands around half-integer multiples of the primary comb $(l \sim 11,22,33$, etc.). The full comb is more complex, and virtually all the modes around the pump at up to $|l| \sim 100$ are excited. The strong influence of the subharmonic higher order comb induces a spectral modulation that has also been observed experimentally in Refs. [6,7]. Such spectrally modulated combs have also been experimentally observed in Ref. [19], in excellent agreement with theory. Further increase of the pump, as in Fig. 10(d), leads to a stronger interaction between the various WGMs. It is noteworthy that in these spectra, the number of photons in the central mode drops to $\left|\mathcal{A}_{0}\right|_{\text {th }}^{2}$ (or $0 \mathrm{~dB}$ ), meaning that all the energy above threshold is distributed among the side modes. These combs can be viewed as energetically optimal. One can also observe that as the pump increases, the oscillating modes of the primary comb globally have a larger order value $|l|$ as the pumping power is increased. This feature is in perfect agreement with the theoretical analysis since the stability diagram of Fig. 8 explains that the stability bands should move away from the central mode as the input power increases.

\section{B. Transient spectral dynamics of the comb}

Investigating the transient dynamics of the combs for a fixed pump power shows how the photons are cascading from the central mode to its neighbors. Understanding this sequence is therefore essential to engineer comb spectra.

Figure 11 displays some snapshot spectra at various instants. At $5 \mu$ s [Fig. 11(a)], the photons from the outside have already filled the central mode. Then, in agreement with our theoretical analysis, it appears that the first modes to be excited belong to the primary comb, as can be seen in Fig. 11(b). One can notice that the modes are excited in a symmetrical manner relative to the pump, thereby indicating that they have been populated through degenerated FWM. At $60 \mu \mathrm{s}$, the spectrum of Fig. 11(c) shows that the central mode interacts with the primary comb to excite an harmonic higher order comb. At $200 \mu \mathrm{s}$ [Fig. 11(d)], a subharmonic higherorder comb emerges and populates the modes that are at middistance from the already existing pattern. Finally, the comb converges toward its steady state [Fig. 11(e)], where almost all the modes are strongly excited, even though a weak spectral modulation is still apparent: This regime is dominated by nondegenerated FWM.

\section{Spectrotemporal representation of the comb dynamics}

It is interesting to monitor the spectrotemporal dynamics of the comb, that is, how the spectrum evolves with time. 


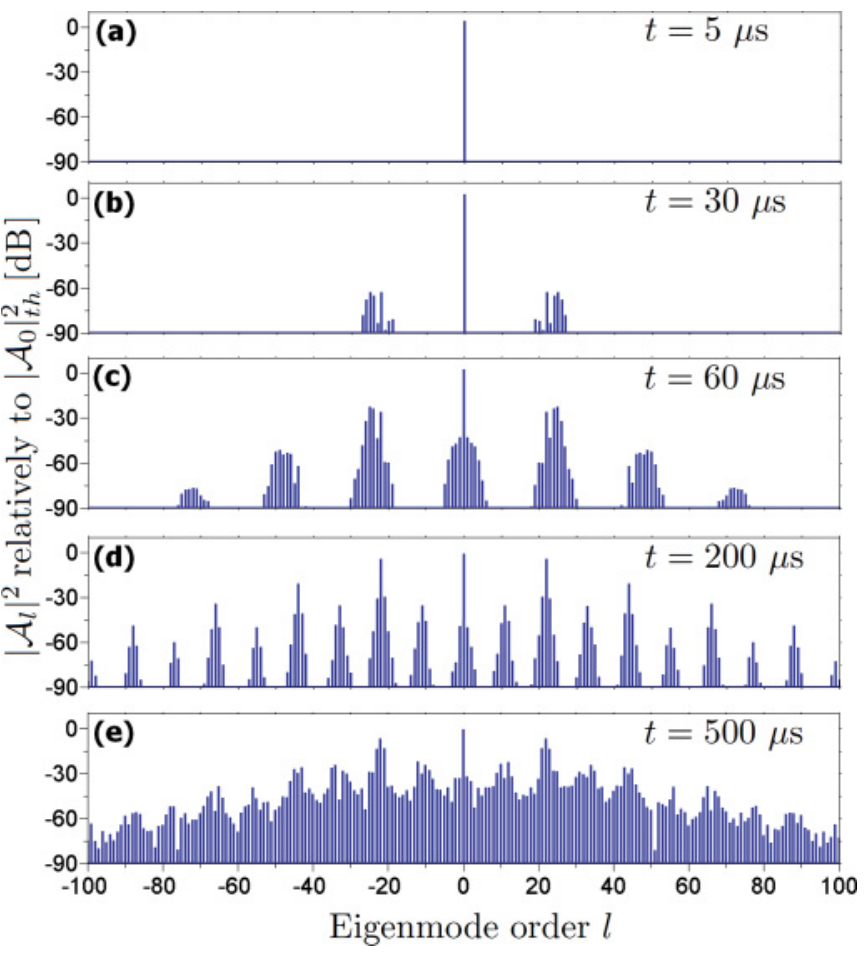

FIG. 11. (Color online) Transient dynamics of the comb when the cavity is pumped with $\left|\mathcal{A}_{0 s}\right|^{2}=1.8\left|\mathcal{A}_{0}\right|_{\text {th }}^{2}$. The parameters are $\zeta=2 \pi \times 400 \mathrm{~Hz}$ and $\sigma=0$.

Figure 12 presents two spectrotemporal diagrams, corresponding to two different pump powers. In Fig. 12(a), the central is pumped 1.2 times above threshold. The steady state
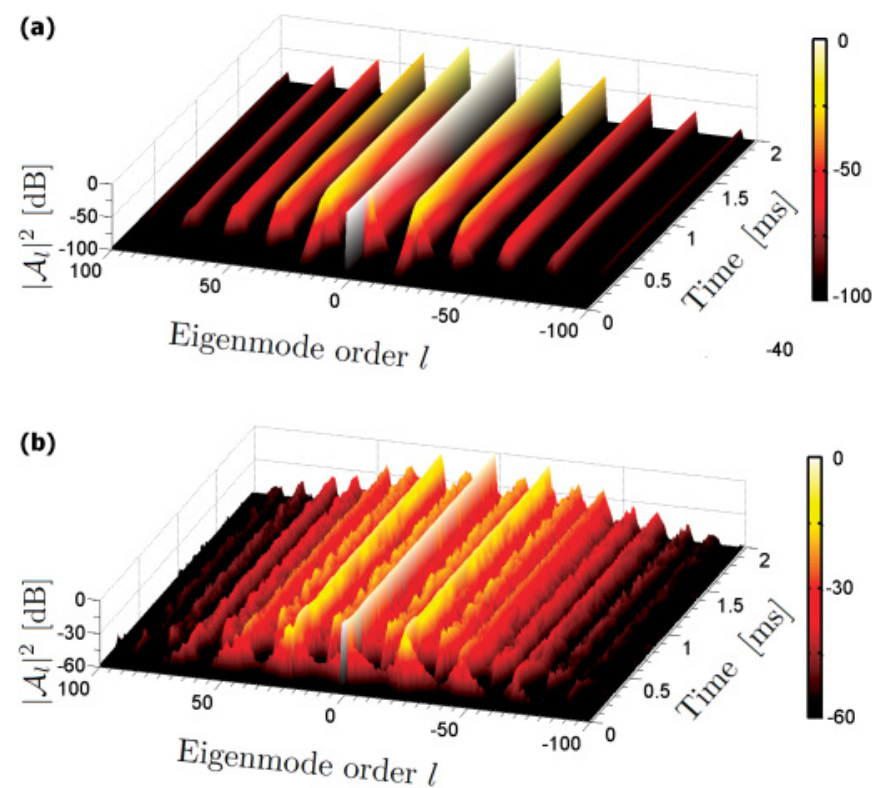

FIG. 12. (Color online) Spectrotemporal behavior of the full comb with $\zeta=2 \pi \times 400 \mathrm{~Hz}$ and $\sigma=0$. (a) $\left|\mathcal{A}_{0 s}\right|^{2}=1.2\left|\mathcal{A}_{0}\right|_{\text {th }}^{2}$, the spectral components converge toward constant values; (b) $\left|\mathcal{A}_{0 s}\right|^{2}=$ $1.8\left|\mathcal{A}_{0}\right|_{\text {th }}^{2}$, the spectrum is chaotic in the time domain. Note that snapshots at $t=2 \mathrm{~ms}$ would correspond to Figs. 10(b) and 10(c), respectively. is stationary, and the oscillating spectral component has a constant amplitude. On the other hand, the central is pumped 1.8 times above threshold in Fig. 12(b), and in this case, it can be seen that the comb becomes nonstationary. In fact, this comb is chaotic with a maximal Lyapunov exponent $\tilde{\lambda} \sim 7 \times$ $10^{4} \mathrm{~s}^{-1}$ (in other words, the temporal dynamics is not predictable beyond $\tilde{\lambda}^{-1} \sim 14 \mu \mathrm{s}$ ). The possibility of a chaotic comb dynamics should be taken into account in future applications because the time-dependent amplitude dynamics of the Kerr modes induces parasite modulation spectral components.

\section{Computational complexity}

A key point in modeling the WGM dynamics is computation time. This aspect gains more importance when the comb is required to span more than one octave [1-3], with several thousands of oscillating modes. Let us consider a comb containing $2 L+1$ modes, with $-L \leqslant l \leqslant L$. It can be demonstrated by recurrence that for any given mode $\eta=l$, the number of resonant four-wave mixing contributions of the kind $\Lambda_{\eta}^{\alpha \beta \mu} \mathcal{A}_{\alpha} \mathcal{A}_{\beta}^{*} \mathcal{A}_{\mu} e^{i \varpi_{\alpha \beta \mu \eta} t}$ is exactly

$$
\aleph(l, L)=3 L^{2}+3 L-l^{2}+1 .
$$

This number is in fact considerable for wide-span combs: In Figs. 10 and 12, for example, the central mode is affected by $\aleph(0,100)=30301$ four-wave mixing terms while the extreme modes still receive $\aleph( \pm 100,100)=20301$ contributions. The computation time $T_{h}$ of each time step is proportional to the sum of the all FWM contributions to all the modes so that

$T_{h} \propto \sum_{l=-L}^{l=L} \aleph(l, L) \simeq \int_{-L}^{L} \aleph(l, L) d l \simeq \frac{16}{3} L^{3}+O\left(L^{2}\right)$.

In other words, the computation time increases in a cubic power fashion with the number of modes. Hence, since simulating a comb with 200 modes requires a few days with a laptop computer, the simulation of an octave-spanning comb with 10000 modes is simply not affordable (computation time is about $50^{3}$ times longer). However, it is known that polynomial algorithms are particularly suited for supercomputing with parallel task distribution. Hence supercomputation can drastically reduce the simulation time and allow for the simulation of these octave-spanning combs. This perspective is particularly interesting because it would enable us to test the dispersion compensation schemes that can potentially push the comb limits to these extremes. It should be emphasized that the computational problem in octave-spanning combs relies exclusively on the number of modes to be simulated: For smaller cavities, the FSR is larger and the number of modes needed to span over one octave is reduced accordingly. For example, a cavity whose diameter is a few tens of micrometers can generate an octave-spanning comb with only few hundreds of modes, whose dynamics can efficiently be simulated with a laptop computer.

\section{EXPANDING THE SPAN OF THE COMB}

As mentioned in the introduction, reaching the comb span beyond one octave allows for the autoreferencing link between optical frequencies and gigahertz microwaves, with 
metrological precision. It is therefore a key objective in comb generation. The model enables us to deduct some interesting pathways that may favor or limit the spectral extension of the comb. For a specific case, we consider the case of a comb spanning from 1000 to $2000 \mathrm{~nm}$, with a central wavelength at $1550 \mathrm{~nm}$, and we try to anticipate the mechanisms that may limit this span according to the model.

A term that seemingly plays an important role is the modal confinement factor $\Gamma_{\eta}$, measuring the geometrical portion of the mode that is within the sphere. It should be noted that though all the WGMs have the same radial order $n=1$, they have different angular orders $\ell$ and therefore different radial profiles, according to Eq. (11). Since the WGMs are strongly confined, this factor is very close to 1 . Moreover, modes with close order $\ell$, also have quasiequal confinement factors; however, this is not the case for modes that are spectrally far away.

The modal linewidth $\Delta \omega_{\eta}$ linearly depends on its central frequency $\omega_{\eta}$, meaning roughly that the bandwidth of the comb peak at $1000 \mathrm{~nm}$ is double the one at $2000 \mathrm{~nm}$ for the same $Q$. Moreover, we also find that the modal linewidth is proportional to the absorption $n_{a}\left(\omega_{\eta}\right)$, which also depends on the frequency. In a $1000 \mathrm{~nm}$ window, the absorption (or equivalently, the transparency) of the bulk medium may vary significantly. For example, absorption typically varies from $2 \times 10^{-5} \mathrm{~cm}^{-1}$ at $1060 \mathrm{~nm}$ to $10^{-3} \mathrm{~cm}^{-1}$ at $2700 \mathrm{~nm}$ for $\mathrm{CaF}_{2}$ [34]. Then, absorption is expected to be stronger at $2000 \mathrm{~nm}$ than at $1000 \mathrm{~nm}$ in the comb, somehow counterbalancing the bandwidth disequilibrium emphasized earlier. The proportionality of $\Delta \omega_{\eta}$ with the confinement factor $\Gamma_{\eta}$ adds a third degree of freedom to this issue.

The ideal resonance condition for FWM, $\varpi_{\alpha \beta \mu \eta}=0$, is not fulfilled because of geometrical and material dispersion [35]. Generally, in the literature, this is considered to be the main mechanism leading to a limited span for the comb. We have shown in this article that anomalous dispersion is needed for comb generation. However, strong dispersion leads to very unequidistant mode distribution, which is incompatible to the energy and momentum conservation requirement of four-wave mixing. Hence an optimal trade-off has to be defined in order to fulfill both constraints. Moreover, the dispersion behavior of the bulk material over an octave is very complex, in particular nonmonotonous, so that it is difficult to anticipate its influence on a general basis.

The FWM coupling also depends on the geometrical overlap of the modes through the factor $\Lambda_{\eta}^{\alpha \beta \mu}$. The modes of a narrow-span comb (close-by values of $\ell$ ) have approximately the same geometrical coupling. Modes that are far away are relatively poorly coupled as their geometrical overlap is not optimal (see Fig. 3). For example, the pumped WGM is geometrically strongly coupled to its adjacent modes but less efficiently coupled to the extreme modes at $1000 \mathrm{~nm}$ than at $2000 \mathrm{~nm}$. This is another feature that should be considered while analyzing the span-limiting mechanisms in opticalfrequency combs. Another important point is that this coupling factor is explicitly frequency dependent because of our normalization in terms of number of photons. This explicit dependence indicates that beyond the spatial overlap, our modal coupling factor also depends on the energy of the photons.

An important issue for octave-spanning combs is the number of oscillating modes. Effectively, an octave with $\mathrm{THz}$ spacing requires only a few hundred modes, while a comb with gigahertz spacing would require more than 10000 . Coupling into the cavity the large amount of photons needed to populate all these modes is a challenge per se. For each cavity, there are physical factors limiting the input pumping power and thus the available number of photons to be transfered to the various side modes. This sets an upper limit to the number of modes that can be excited above a given level and impedes the generation of arbitrarily wide combs with arbitrarily narrow FSR spacings.

\section{CONCLUSION}

We have established a general framework for the study of optical-frequency combs generated with monolithic resonators. Starting with a wave equation, we have constructed on a semiclassical basis a set of coupled time-domain differential equations ruling the dynamics of the electric field for each cavity mode. We have provided formulas for the threshold number of photons needed for comb generation as well a method to predict the first side modes that will be excited by the pump. Our analysis has shown that even though strong dispersion is naturally detrimental to comb generation, weak anomalous dispersion is needed in order to lift the degeneracy between the unstable hysteresis area for the central mode and the stable area for side mode excitation in the parametric space.

The theoretical analysis has demonstrated that the cascading mechanism leading to a wide-span comb is complex, as the pump preferably excites a limited set of modes (the primary comb) and then utilizes them as relays to excite the remaining side modes through a thresholdless bichromatic pumping process. It was shown that multiple FSR spacing and spectral comb modulation are a consequence of this sequential excitation scenario originating from the interplay between anomalous dispersion and Kerr nonlinearity. Opticalfrequency comb generation in this context presents strong similarities with modulational instability and supercontinuum generation [36].

Beyond the study of optical-frequency combs, this modal approach could be useful to investigate other nonlinear phenomena in these WGM resonators such as erbium-doped lasers [37-41], Raman lasers [42,43], and Brillouin lasers [44]. More generally, this work is integrated to the perspective of nonlinear phenomena in optical cavities and waveguides, and it is a fruitful paradigm for crystalline or silicon photonics [45-47]. It is noteworthy that a quantification of this model would also enable the exploration of many interesting quantum electrodynamic effects $[48,49]$.

Though it is very insightful and instructive, our modal approach can still be improved. Particular attention should be paid to this issue of thermal effects, even though it may appear at first sight to be a technical issue. For example, our model relies on the so-called cold-cavity mode expansion, where we assume that the refraction index is temperature independent. However, as shown in Fig. 2, the energy of WGMs is confined in a very narrow torus inside the cavity, and this high density of energy leads to a temperature-induced local change in the refraction index, sometimes referred to as thermal lensing. This thermal lensing induces a temperature-dependent shift in the eigenmode distribution, linewidths, and spatial extension. 
Moreover, it interacts in a very complex way with the modal dynamics of the system [50]. Further investigations including the temperature variable in our equations are needed in order to know if thermal lensing plays a positive or a negative role as far as the spectral extension of the comb is concerned. Along the same line, the WGMs also experience Kerr lensing as the optical power also modifies locally the refraction index and thereby the various physical properties of the WGMs (mainly spectrum, modal linewidths, and spatial extensions).

We also know that generally, waveguides are not spherical (the principal and curvature radii are different). Hence the spherical eigenmodes $\boldsymbol{\Upsilon}_{\eta}(\mathbf{r})$ are no longer (nearly) orthogonal, and spurious coupling terms between all modes should be taken into account in our equations after Hermitian projection. While studying ultrawide combs, it may also be possible that the modal frequencies $\omega_{\eta}$ should be replaced by the instantaneous frequencies $\omega_{\eta}-i \dot{\mathcal{E}}_{\eta} / \mathcal{E}_{\eta}$ and that the second derivative terms $\ddot{\mathcal{E}}_{\eta}$ may be no longer negligible.

A straightforward step after our completely deterministic study is to include the effect of noise. The study of stochastic equations within the frame of Langevin equations would be an interesting tool to investigate the optical phase diffusion of each mode but also the phase noise of the microwaves that can be extracted from these combs. Another issue that would deserve attention is the temporal output of the system:
Effectively, the generation of a periodic train of pulses is expected, but the various comb configurations (spectral modulation, multiple FSR spacing, etc.) could induce a wide variety of output wave forms that may be particularly interesting from the perspective of optical pulse synthesis. Various configurations with multipolarized or polychromatic pumping schemes [33] can also be investigated with this modal approach, and we anticipate that interesting phenomena may arise from these degrees of freedom. This system is also very interesting as it enables us to understand the nonlinear dynamics of high-dimensional systems coupled through a single reservoir of energy. Finally, the most important prospective efforts will be devoted to the achievement of an ultrawide comb spanning beyond one octave, with the smallest FSR spacing possible.

\section{ACKNOWLEDGMENTS}

This work was performed at the Jet Propulsion Laboratory, California Institute of Technology, under a contract with NASA. Y.K.C. acknowledges support from the NASA postdoctoral program, administered by Oak Ridge Associated Universities (ORAU). The authors would also like to acknowledge logistic support from the JPL Supercomputing and Visualization Facility.
[1] S. T. Cundiff and J. Ye, Rev. Mod. Phys. 75, 325 (2003).

[2] J. L. Hall, Rev. Mod. Phys. 78, 1279 (2006).

[3] T. W. Hänsch, Rev. Mod. Phys. 78, 1297 (2006).

[4] P. Del'Haye, A. Schliesser, O. Arcizet, T. Wilken, R. Holzwarth, and T. J. Kippenberg, Nature (London) 450, 1214 (2007).

[5] P. Del Haye, O. Arcizet, A. Schliesser, R. Holzwarth, and T. J. Kippenberg, Phys. Rev. Lett. 101, 053903 (2008).

[6] A. A. Savchenkov, A. B. Matsko, V. S. Ilchenko, I. Solomatine, D. Seidel, and L. Maleki, Phys. Rev. Lett. 101, 093902 (2008).

[7] I. S. Grudinin, N. Yu, and L. Maleki, Opt. Lett. 34, 878 (2009).

[8] A. N. Oraevsky, Quantum Electron. 32, 377 (2002).

[9] A. B. Matsko and V. S. Ilchenko, IEEE J. Sel. Top. Quantum Electron. 12, 3 (2006).

[10] V. S. Ilchenko and A. B. Matsko, IEEE J. Sel. Top. Quantum Electron. 12, 15 (2006).

[11] I. S. Grudinin, A. B. Matsko, A. A. Savchenkov, D. Strekalov, V. S. Ilchenko, and L. Maleki, Opt. Commun. 265, 33 (2006).

[12] T. J. Kippenberg, Ph.D. thesis, California Institute of Technology, 2004.

[13] K. Vahala, ed., Optical Microcavities (World Scientific, Singapore, 2004).

[14] T. J. Kippenberg, S. M. Spillane, and K. J. Vahala, Phys. Rev. Lett. 93, 083904 (2004).

[15] A. A. Savchenkov, A. B. Matsko, D. Strekalov, M. Mohageg, V. S. Ilchenko, and L. Maleki, Phys. Rev. Lett. 93, 243905 (2004).

[16] A. B. Matsko, A. A. Savchenkov, D. Strekalov, V. S. Ilchenko, and L. Maleki, Phys. Rev. A 71, 033804 (2005).

[17] I. H. Agha, Y. Okawachi, M. A. Foster, J. E. Sharping, and A. L. Gaeta, Phys. Rev. A 76, 043837 (2007).

[18] I. H. Agha, Y. Okawachi, and A. L. Gaeta, Opt. Express 17, 16209 (2009).
[19] Y. K. Chembo, D. V. Strekalov, and N. Yu, Phys. Rev. Lett. 104, 103902 (2010).

[20] B. R. Johnson, J. Opt. Soc. Am. A 10, 343 (1993).

[21] S. Schiller, Appl. Opt. 32, 2181 (1993).

[22] H. M. Lai, P. T. Leung, K. Young, P. W. Barber, and S. C. Hill, Phys. Rev. A 41, 5187 (1990).

[23] C. C. Lam, P. T. Leung, and K. Young, J. Opt. Soc. Am. B 9 , 1585 (1992).

[24] M. L. Gorodetsky and V. S. Ilchenko, J. Opt. Soc. Am. B 16, 147 (1997).

[25] A. Yariv, Electron. Lett. 36, 321 (2000).

[26] Y. Dumeige, S. Trebaol, L. Ghisa, T. K. N. Nguyen, H. Tavernier, and P. Féron, J. Opt. Soc. Am. B 25, 2073 (2008).

[27] I. H. Malitson, Appl. Opt. 2, 1103 (1963).

[28] M. Daimon and A. Masumura, Appl. Opt. 41, 5275 (2002).

[29] A. A. Savchenkov, E. Rubiola, A. B. Matsko, V. S. Ilchenko, and L. Maleki, Opt. Express 16, 4130 (2008).

[30] M. M. Mazumder, S. C. Hill, D. Q. Chowdhury, and R. K. Chang, J. Opt. Soc. Am. B 12, 297 (1995).

[31] Y. K. Chembo, L. Larger, R. Bendoula, and P. Colet, Opt. Express 16, 9067 (2008).

[32] Y. K. Chembo, S. K. Mandre, I. Fischer, W. Elsässer, and P. Colet, Phys. Rev. A 79, 013817 (2009).

[33] D. V. Strekalov and N. Yu, Phys. Rev. A 79, 041805 (2009).

[34] Corning Inc. (unpublished).

[35] V. S. Ilchenko, A. A. Savchenkov, A. B. Matsko, and L. Maleki, J. Opt. Soc. Am. A 20, 157 (2003).

[36] J. M. Dudley, G. Genty, and S. Coen, Rev. Mod. Phys. 78, 1135 (2006).

[37] F. Lissillour, P. Féron, N. Dubreuil, P. Dupriez, M. Poulain, and G. M. Stephan, Electron. Lett. 36, 1382 (2000). 
[38] F. Lissillour, D. Messager, G. Stéphan, and P. Féron, Opt. Lett. 26, 1051 (2001).

[39] B. Min, T. J. Kippenberg, L. Yang, K. J. Vahala, A. Polman, and J. Kalkman, Phys. Rev. A 70, 033803 (2004).

[40] T. J. Kippenberg, J. Kalkman, A. Polman, and K. J. Vahala, Phys. Rev. A 74, 051802 (2006).

[41] J. M. Ward, P. Féron, and S. N. Chormaic, IEEE Photonics Technol. Lett. 20, 392 (2008).

[42] S. M. Spillane, T. J. Kippenberg, and K. J. Vahala, Nature (London) 415, 621 (2002).

[43] T. J. Kippenberg, S. M. Spillane, D. K. Armani, and K. J. Vahala, Opt. Lett. 29, 1224 (2004).

[44] I. S. Grudinin, A. B. Matsko, and L. Maleki, Phys. Rev. Lett. 102, 043902 (2009).
[45] Q. Lin, O. J. Painter, and G. P. Agrawal, Opt. Express 15, 16604 (2007).

[46] J. S. Levy, A. Gondarenko, M. A. Foster, A. C. TurnerFoster, A. L. Gaeta, and M. Lipson, Nature Photon. 4, 37 (2009).

[47] A. Chiasera, Y. Dumeige, P. Féron, M. Ferrari, Y. Jestin, G. Nunzi Conti, S. Pelli, S. Soria, and G. C. Righini, Laser Photon. Rev. 4, 457 (2010).

[48] K. Vahala, Nature (London) 424, 839 (2003).

[49] S. M. Spillane, T. J. Kippenberg, K. J. Vahala, K. W. Goh, E. Wilcut, and H. J. Kimble, Phys. Rev. A 71, 013817 (2005).

[50] A. E. Fomin, M. L. Gorodetsky, I. S. Grudinin, and V. S. Ilchenko, J. Opt. Soc. Am. B 22, 459 (2005). 